\title{
Strangeness in compact stars
}

\author{
Fridolin Weber ${ }^{* \dagger}$ \\ Department of Physics, San Diego State University \\ E-mail: fweber@sciences.sdsu.edu
}

\section{Andreu Torres i Cuadrat}

Physics Department, Universitat Autonoma de Barcelona

E-mail: andreu.torres@uab.es

\section{Alexander Ho}

Department of Physics, San Diego State University

E-mail: aho@rohan.sdsu.edu

\section{Philip Rosenfield}

Department of Astronomy, San Diego State University

E-mail: philrose@sciences.sdsu.edu

\begin{abstract}
Astrophysicists distinguish between three different types of compact stars. These are white dwarfs, neutron stars, and black holes. The former contain matter in one of the densest forms found in the Universe. This feature, together with the unprecedented progress in observational astronomy, makes such stars superb astrophysical laboratories for a broad range of exciting physical studies. This article studies the role of strangeness for compact star phenomenology. Strangeness is carried by hyperons, mesons, H-dibaryons, and strange quark matter, and may leave its mark in the masses, radii, cooling behavior, surface composition and the spin evolution of compact stars.
\end{abstract}

29th Johns Hopkins Workshop on current problems in particle theory: strong matter in the heavens

\section{1-3 August \\ Budapest}

\footnotetext{
* Speaker.

${ }^{\dagger}$ Supported by the National Science Foundation under Grant PHY-0457329, and by the Research Corporation.
} 


\section{Introduction}

Astrophysicists distinguish between three different types of compact stellar objects. These are white dwarfs, neutron stars, and black holes. The latter constitute a region of space which has so much mass-energy concentrated in it that no particles (not even light) inside the black hole's event horizon can escape the black hole's gravitational pull. The situation is very different for neutron stars and white dwarfs, which are about as massive as the sun (mass $M_{\odot}=2 \times 10^{30} \mathrm{~kg}$ ) but whose radii are much smaller than the sun's radius $\left(R_{\odot}=7 \times 10^{5} \mathrm{~km}\right)$. Model calculations predict that the matter in the cores of neutron stars is compressed to densities ranging from a few times the density of an atomic nucleus, $2.5 \times 10^{14} \mathrm{~g} / \mathrm{cm}^{3}$, to densities that may be ten to twenty times higher $[1,2,3]$. In comparison to that, white dwarfs of average mass, $M \sim 0.6 M_{\odot}$ are at least by a factor of $10^{7}$ less dense than neutron stars. The tremendous densities (and thus pressures) existing inside neutron stars make them superb astrophysical laboratories for a wide range of fascinating physical studies $[1,2,4]$. These include the exploration of nuclear processes in an environment extremely rich of electrons and neutrons, and the formation of new states of matter, like quark matter which is being sought at the most powerful terrestrial particle colliders. If quark matter exists in the cores of neutron stars, it will be a color superconductor whose condensation pattern has been shown to be very complex $[5,6,7]$. It has also been theorized that quark matter (known as strange quark matter) may be even more stable than atomic nuclei. In the latter event neutron stars should be entirely made of strange quark matter, possibly enveloped in a very thin nuclear crust. Such objects are called strange stars $[8,9,10]$. Strangeness, therefore, carried by hyperons, mesons, H-dibaryons, and strange quark matter, plays a key role for compact star physics and phenomenology, as will be discussed in this paper (see Ref. [4] for a detailed recent review on this topic).

\section{Composition of high-density neutron star matter and EoS}

The properties of neutron stars are determined by the equation of state (EoS) of neutron star matter. The EoS of neutron star matter below neutron drip, which occurs at densities around $4 \times 10^{11} \mathrm{~g} / \mathrm{cm}^{3}$, and at densities above neutron drip but below the saturation density of nuclear matter is relatively well known. This is to a less extent the case for the EoS in the vicinity of the saturation density of normal nuclear matter, $n_{0}=0.16 \mathrm{fm}^{-3}$ (energy density of $\varepsilon_{0}=140 \mathrm{MeV} / \mathrm{fm}^{3}$ ). Finally, the physical properties of matter at still higher densities are extremely uncertain so that the associated EoS is only very poorly known $[1,2,3]$. This is graphically indicated by the hatched areas in Fig. 1, which is based on three Walecka-type models for the EoS. The HV model is a relativistic non-linear mean-field equation of state computed for nucleons $(\mathrm{N}=n, p)$ and hyperons $(H=\Sigma, \Lambda, \Xi)$ in chemical equilibrium [4, 11]. The other two models, $\mathrm{G}_{300}^{\mathrm{B} 180}$ [1] and CFL [12], account additionally for the presence of up, down, and strange quarks in neutron star matter. The quarks are treated as normal unpaired quarks $(\mathrm{Q})$ in the $\mathrm{G}_{300}^{\mathrm{B} 180}$ model, and as color-flavor locked superconducting quarks (sQ) with a superfluid gap of $\Delta=100 \mathrm{MeV}$ in the CFL model. The model labeled DD-RBHF is a density dependent relativistic Brueckner-Hartree-Fock EoS which accounts for nucleons and hyperons [13, 14]. It is obvious from Fig. 1 that, depending on stellar composition, neutron star properties such as masses, radii, moments of inertia, redshifts, or limiting rotational periods may vary significantly with strangeness. 


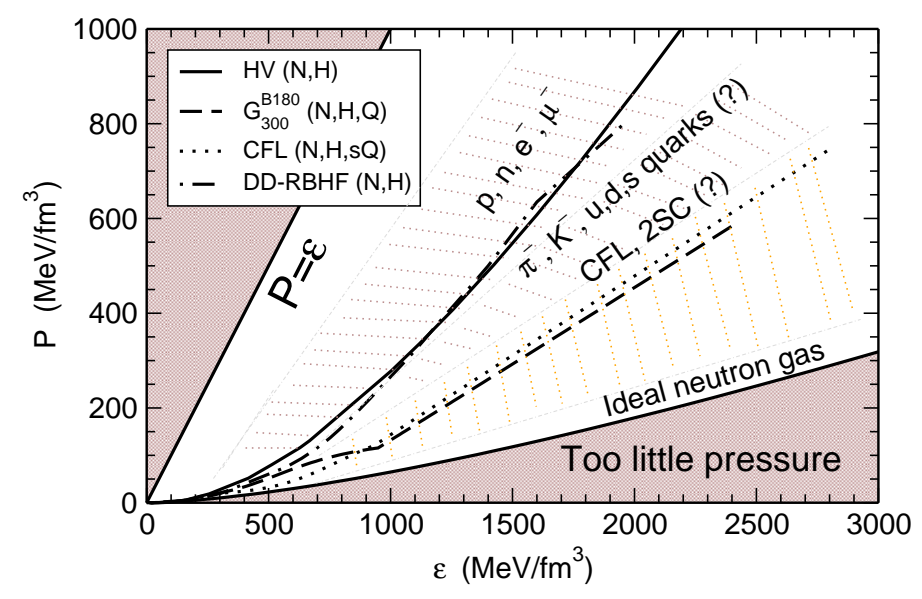

Figure 1: Models for the EoS of neutron star matter.

\subsection{Hyperons}

At the densities in the interior of neutron stars, the neutron chemical potential, $\mu^{n}$, is likely to exceeds the masses, modified by interactions, of $\Sigma, \Lambda$ and possibly $\Xi$ hyperons [11]. Hence, in addition to nucleons, neutron star matter is expected to have significant populations of hyperons and possibly even $\Delta$ 's [15]. If so, pure neutron matter would constitute an excited state relative to hyperonic (many-baryon) matter which, therefore, would quickly transform via weak reactions like

$$
n \rightarrow p+e^{-}+\bar{v}_{e}
$$

to the lower energy state. The chemical potentials associated with reaction (2.1) in equilibrium obey the relation

$$
\mu^{n}=\mu^{p}+\mu^{e^{-}}
$$

where $\mu^{\bar{v}_{e}}=0$ since the mean free path of (anti) neutrinos is much smaller than the radius of neutron stars. Hence (anti) neutrinos do not accumulate inside neutron stars. This is different for hot proto-neutron stars [16]. Equation (2.2) is a special case of the general relation

$$
\mu^{\chi}=B^{\chi} \mu^{n}-q^{\chi} \mu^{e^{-}},
$$

which holds in any system characterized by two conserved charges. These are in the case of neutron star matter electric charge, $q^{\chi}$, and baryon number charge, $B^{\chi}$. Application of Eq. (2.3) to the $\Lambda$ hyperon $\left(B^{\Lambda}=1, q^{\Lambda}=0\right)$, for instance, leads to

$$
\mu^{\Lambda}=\mu^{n} .
$$

Ignoring particle interactions, the chemical potential of a relativistic particle of type $\chi$ is given by

$$
\mu^{\chi}=\omega\left(k_{F_{\chi}}\right) \equiv \sqrt{m_{\chi}^{2}+k_{F_{\chi}}^{2}}
$$

where $\omega\left(k_{F_{\chi}}\right)$ is the single-particle energy of the particle and $k_{F_{\chi}}$ its Fermi momentum. Substituting (2.5) into (2.4) leads to

$$
k_{F_{n}} \geq \sqrt{m_{\Lambda}^{2}-m_{n}^{2}} \simeq 3 \mathrm{fm}^{-1} \Rightarrow n \equiv \frac{k_{F_{n}}{ }^{3}}{3 \pi^{2}} \simeq 2 n_{0},
$$


where $m_{\Lambda}=1116 \mathrm{MeV}$ and $m_{n}=939 \mathrm{MeV}$ was used. That is, if interactions among the particles are ignored, neutrons are replaced with $\Lambda$ 's in neutron star matter at densities as low as two times the density of nuclear matter. This result is only slightly altered by the inclusion of particle interactions [11]. Aside from chemical equilibrium, the condition of electric charge neutrality of neutron star matter,

$$
\sum_{\chi=p, \Sigma^{ \pm}, \Xi^{-}, \Delta^{++}, \ldots ; e^{-}, \mu^{-}} q^{\chi} k_{F_{\chi}}^{3}+3 \pi^{2} n^{M} \Theta\left(\mu^{M}-m_{M}\right) \equiv 0,
$$

where $M$ stands for $\pi^{-}$or $K^{-}$mesons, plays a key role for the particle composition of neutron star matter too. The last term in (2.7) accounts for the possible existence of either a $\pi^{-}$or a $K^{-}$meson condensate in neutron star matter, which will be discussed in more detail in Sect. 2.2 below. Before, however, we illustrate the importance of Eqs. (2.2) and (2.7) for the proton-neutron fraction of neutron star matter. The beta decay and electron capture processes among nucleons, $n \rightarrow$ $p+e^{-}+\bar{v}_{e}$ and $p+e^{-} \rightarrow n+v_{e}$ respectively, also known as nucleon direct Urca processes, are only possible in neutron star matter if the proton fraction exceeds a certain critical value [17]. Otherwise energy and momentum can not be conserved simultaneously for these reactions so that they are forbidden. For a neutron star made up of only nucleons and electrons, it is rather straightforward to show that the critical proton fraction is around $11 \%$. This follows from $\mathbf{k}_{F_{n}}=\mathbf{k}_{F_{p}}+\mathbf{k}_{F_{e}}$ combined with the condition of electric charge neutrality of neutron star matter. The triangle inequality then requires for the magnitudes of the particle Fermi momenta $k_{F_{n}} \leq k_{F_{p}}+k_{F_{e}}$, and charge neutrality dictates that $k_{F_{p}}=k_{F_{e}}$. Substituting $k_{F_{p}}=k_{F_{e}}$ into the triangle inequality leads to $k_{F_{n}} \leq 2 k_{F_{p}}$ so that for the particle number densities of neutrons and protons $n_{n} \leq 8 n_{p}$. Expressed as a fraction of the system's total baryon number density, $n \equiv n_{p}+n_{n}$, one thus arrives at $n_{p} / n>1 / 9 \simeq 0.11$, which is the figure quoted just above. Medium effects and interactions among the particles modify this value only slightly but the presence of muons raises it to about 0.15 . Hyperons, which may exist in neutron star matter rather abundantly, produce neutrinos via direct Urca processes like $\Sigma^{-} \rightarrow \Lambda+e^{-}+\bar{v}_{e}$ and $\Lambda+e^{-} \rightarrow \Sigma^{-}+v_{e}[18]$. The direct Urca processes are of key importance for neutron star cooling, which will be discussed briefly in Sect. 6 . In most cases, the nucleon direct Urca process is more efficient than the ones involving hyperons [19, 20].

\subsection{Meson condensates}

The pion or kaon meson fields may develop condensates in dense neutron star matter. These condensates would have two important effects on neutron stars. Firstly, they would soften the EoS above the critical density for onset of condensation, which reduces the maximum neutron star mass. Secondly, since the $\left\langle\pi^{-}\right\rangle$or $\left\langle K^{-}\right\rangle$condensates can absorb as little or as much momentum as required by the scattering processes $n+<\pi^{-}>\rightarrow n+e^{-}+\bar{v}_{e}$ or $n+<K^{-}>\rightarrow n+e^{-}+\bar{v}_{e}$, the associated neutrino emissivities are very high which leads to fast neutron star cooling [20, 21] (see Sect. 6). Since the $K^{-}$condensate process involves a change in strangeness, it is roughly by a factor $\sin ^{2} \theta_{C} \simeq 1 / 20\left(\theta_{C}\right.$ denotes the Cabibbo angle) less efficient than the $\pi^{-}$condensate process. However, medium effects can reduce the impact of the $\pi^{-}$condensate on stellar cooling by about one order of magnitude, making it comparable to the efficiency of the $K^{-}$condensate. Estimates predict the onset of $\pi^{-}$condensation at densities around $n^{\pi} \sim 2 n_{0}$, with $n_{0}=0.16 \mathrm{fm}^{-3}$ the empirical nuclear matter density. However, this density is very sensitive to the strength of the effective nucleon particle-hole repulsion in the isospin $T=1$, spin $S=1$ channel, which tends to 
suppress $\pi^{-}$condensation and may push $n^{\pi}$ to much higher values. Similarly, depending on the nuclear model, the threshold density for the onset of $K^{-}$condensation, $n^{K}$, is probably at least as high as $4 n_{0}[22,23]$.

$K^{-}$condensation can only occur in neutron star matter if the electron chemical potential equals the effective in-medium meson mass, according to the schematic reaction $e^{-} \rightarrow K^{-}+v_{e}$, with the neutrinos leaving the star (see Fig. 2). This reaction would be followed by $n \rightarrow p+K^{-}$. By this

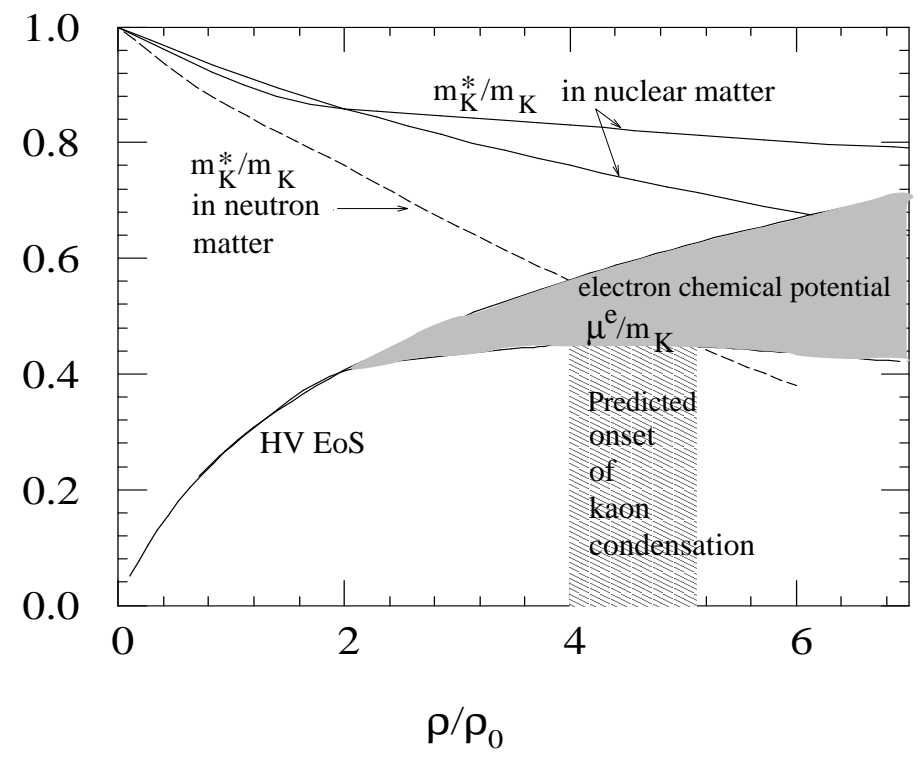

Figure 2: The effective kaon mass, $m_{K}^{*}$, in nuclear matter and neutron star matter [4]. Data taken from [24] and [22], respectively.

conversion the nucleons in the cores of newly formed neutron stars can become half neutrons and half protons [25]. The relatively isospin symmetric composition achieved in this way resembles the one of isospin symmetric atomic nuclei, which are made up of equal numbers of neutrons and protons. Neutron star matter is therefore referred to in this picture as nucleon matter, and neutron stars constructed for such an EoS are referred to as nucleon stars [25, 26, 27, 28].

\subsection{H-dibaryons}

A novel particle that could make its appearance in the center of a neutron star is the so-called H-dibaryon, a doubly strange six-quark composite with spin and isospin zero, and baryon number two [29]. Since its first prediction in the 1970s, the H-dibaryon has been the subject of many theoretical and experimental studies as a possible candidate for a strongly bound exotic state. In neutron stars, which may contain a significant fraction of $\Lambda$ hyperons, the $\Lambda$ 's could combine to form H-dibaryons, which could give way to the formation of $\mathrm{H}$-dibaryon matter at densities somewhere above $\sim 3 \varepsilon_{0}[30,31,32]$ depending on the in-medium properties of the H-dibaryon. For an attractive optical potential, $U_{\mathrm{H}}$, of the H-dibaryon at normal nuclear density the equation of state is softened considerably, as shown in Fig. 3. H-dibaryon matter could thus exist in the cores of moderately dense neutron stars. H-dibaryons with a vacuum mass of about $2.2 \mathrm{GeV}$ and 


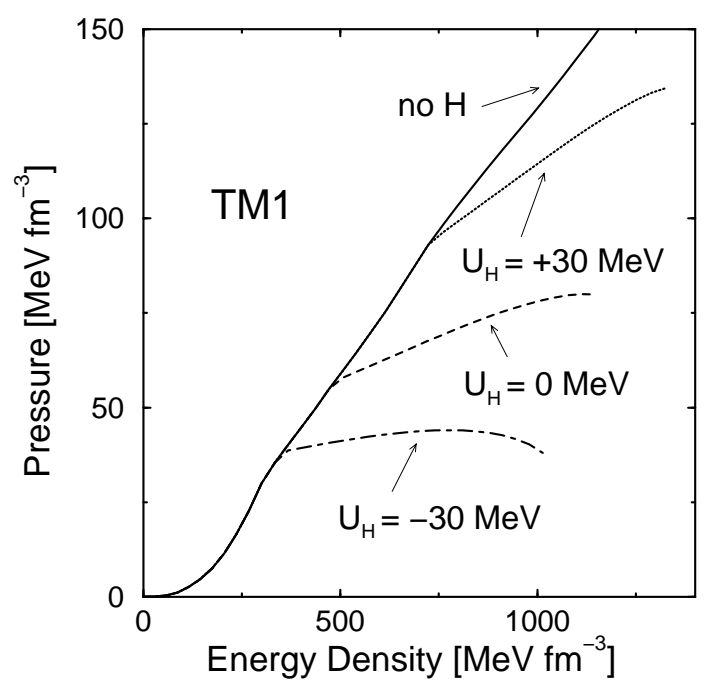

Figure 3: EoS of neutron star matter accounting for a H-dibaryon condensate [32]. $U_{\mathrm{H}}$ is the optical potential of the H-dibaryon at normal nuclear density.

a moderately attractive potential in the medium of about $U_{\mathrm{H}}=-30 \mathrm{MeV}$, for instance, could go into a boson condensate in the cores of neutron stars if the limiting star mass is about that of the Hulse-Taylor pulsar PSR 1913+16, $M=1.444 M_{\odot}$ [32]. Conversely, if the medium potential were moderately repulsive, around $U_{\mathrm{H}}=+30 \mathrm{MeV}$, the formation of H-dibaryons may only take place in heavier neutron stars of masses greater than about $1.6 M_{\odot}$. If formed, however, H-matter may not remain dormant in neutron stars but, because of its instability against compression, could trigger the conversion of neutron stars into hypothetical strange stars [31, 33, 34].

\subsection{Quark deconfinement}

One item that came recently into particular focus concerns the possible existence of quark matter in the cores of neutron stars $[2,5,6]$. The phase transition from confined hadronic matter to deconfined quark matter is characterized by the conservation of baryon charge and electric charge. The Gibbs condition for phase equilibrium then is that the two associated chemical potentials, $\mu^{n}$ and $\mu^{e}$, and the pressure in the two phases be equal $[1,35]$,

$$
P_{\mathrm{H}}\left(\mu^{n}, \mu^{e},\{\chi\}, T\right)=P_{\mathrm{Q}}\left(\mu^{n}, \mu^{e}, T\right) .
$$

$P_{\mathrm{H}}$ denoted the pressure of hadronic matter computed for a given hadronic matter Lagrangian $\mathscr{L}_{\mathrm{M}}(\{\chi\})$, where $\{\chi\}$ denotes the field variables and Fermi momenta that characterize a solution to the field equations of confined hadronic matter,

$$
\begin{aligned}
\left(i \gamma^{\mu} \partial_{\mu}-m_{\chi}\right) \psi_{\chi}(x) & =\sum_{M=\sigma, \omega, \pi, \ldots} \Gamma_{M \chi} M(x) \psi_{\chi}(x), \\
\left(\partial^{\mu} \partial_{\mu}+m_{\sigma}^{2}\right) \sigma(x) & =\sum_{\chi=p, n, \Sigma, \ldots} \Gamma_{\sigma \chi} \bar{\psi}_{\chi}(x) \psi_{\chi}(x),
\end{aligned}
$$

plus additional equations for the other meson fields $(M=\omega, \pi, \rho, \ldots)$. The pressure of quark matter, $P_{\mathrm{Q}}$, is obtainable from the bag model. The quark chemical potentials $\mu^{u}, \mu^{d}, \mu^{s}$ are related to the 

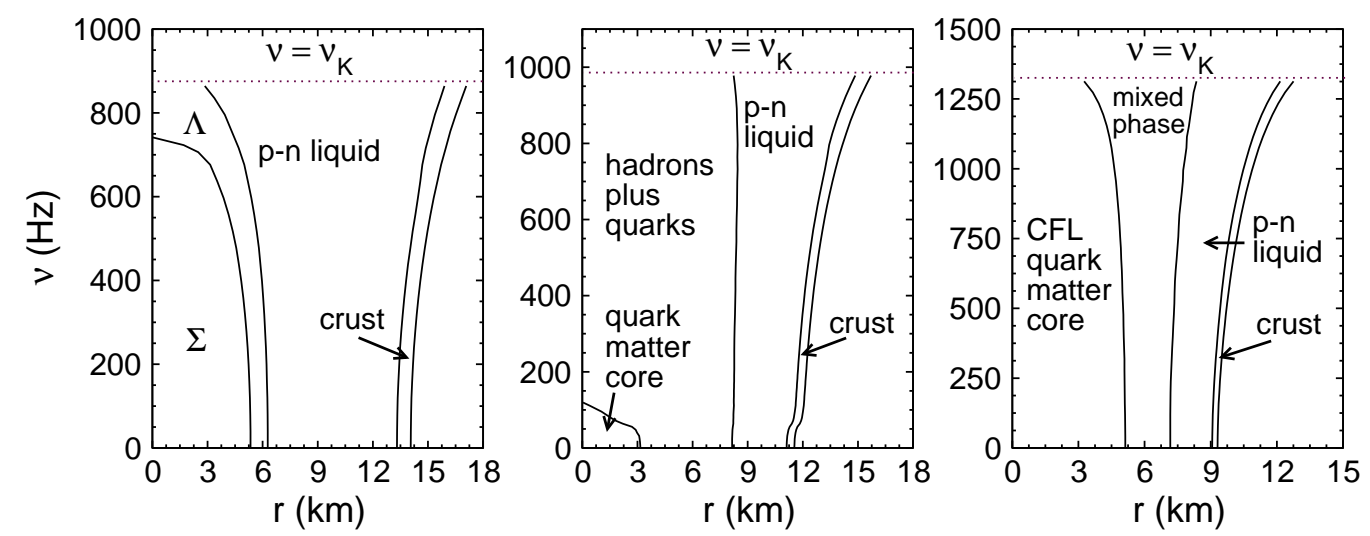

Figure 4: Dependence of stellar compositions on neutron star spin frequency, $v$, for the $\mathrm{HV}, \mathrm{G}_{300}^{\mathrm{B} 180}$, and CFL (from left to right) EoSs. The non-rotating stellar mass in each case is about $1.4 M_{\odot} . v_{\mathrm{K}}$ denotes the Kepler (mass-shedding) frequency of each sequence, discussed in Sect. 4.

baryon and charge chemical potentials as

$$
\mu^{u}=\frac{1}{3} \mu^{n}-\frac{2}{3} \mu^{e}, \quad \mu^{d}=\mu^{s}=\frac{1}{3} \mu^{n}+\frac{1}{3} \mu^{e} .
$$

Equation (2.8) is to be supplemented with the two global relations for conservation of baryon charge and electric charge within an unknown volume $V$ containing $A$ baryons. The first one is given by

$$
n \equiv \frac{A}{V}=(1-\eta) n_{\mathrm{H}}\left(\mu^{n}, \mu^{e}, T\right)+\eta n_{\mathrm{Q}}\left(\mu^{n}, \mu^{e}, T\right),
$$

where $\eta \equiv V_{\mathrm{Q}} / V$ denotes the volume proportion of quark matter, $V_{\mathrm{Q}}$, in the unknown volume $V$, and $n_{\mathrm{H}}$ and $n_{\mathrm{Q}}$ are the baryon number densities of hadronic matter and quark matter. Global neutrality of electric charge within the volume $V$ can be written as

$$
0=\frac{Q}{V}=(1-\eta) q_{\mathrm{H}}\left(\mu^{n}, \mu^{e}, T\right)+\eta q_{\mathrm{Q}}\left(\mu^{n}, \mu^{e}, T\right)+q_{\mathrm{L}},
$$

with $q_{i}$ the electric charge densities of hadrons, quarks, and leptons. For a given temperature, $T$, Eqs. (2.8) to (2.13) serve to determine the two independent chemical potentials and the volume $V$ for a specified volume fraction $\eta$ of the quark phase in equilibrium with the hadronic phase. After completion $V_{\mathrm{Q}}$ is obtained as $V_{\mathrm{Q}}=\eta V$. Because of Eqs. (2.8) through (2.13) the chemical potentials depend on the proportion $\eta$ of the phases in equilibrium, and hence so also all properties that depend on them, i.e. the energy densities, baryon and charge densities of each phase, and the common pressure. For the mixed phase, the volume proportion of quark matter varies from $0 \leq \eta \leq 1$ and the energy density is the linear combination of the two phases [1,35],

$$
\varepsilon=(1-\eta) \varepsilon_{\mathrm{H}}\left(\mu^{n}, \mu^{e},\{\chi\}, T\right)+\eta \varepsilon_{\mathrm{Q}}\left(\mu^{n}, \mu^{e}, T\right) .
$$

Hypothetical neutron star compositions computed along the lines described above are shown in Fig. 4. Possible astrophysical signals originating from quark deconfinement will be discussed in Sect. 5 [1, 2, 36, 37]. 


\subsection{Color superconductivity of quark matter}

There has been much recent progress in our understanding of quark matter, culminating in the discovery that if quark matter exists it ought to be in a color superconducting state $[5,6,38,39]$. This is made possible by the strong interaction among the quarks which is very attractive in some channels. Pairs of quarks are thus expected to form Cooper pairs very readily. Since pairs of quarks cannot be color neutral, the resulting condensate will break the local color symmetry and form what is called a color superconductor. The phase diagram of such matter is expected to be very complex $[5,6]$. This is caused by the fact that quarks come in three different colors, different flavors, and different masses. Moreover, bulk matter is neutral with respect to both electric and color charge, and is in chemical equilibrium under the weak interaction processes that turn one quark flavor into another. To illustrate the condensation pattern briefly, we note the following pairing ansatz for the quark condensate [40],

$$
\left\langle\psi_{f_{a}}^{\alpha} C \gamma_{5} \psi_{f_{b}}^{\beta}\right\rangle \sim \Delta_{1} \varepsilon^{\alpha \beta 1} \varepsilon_{f_{a} f_{b} 1}+\Delta_{2} \varepsilon^{\alpha \beta 2} \varepsilon_{f_{a} f_{b} 2}+\Delta_{3} \varepsilon^{\alpha \beta 3} \varepsilon_{f_{a} f_{b} 3},
$$

where $\psi_{f_{a}}^{\alpha}$ is a quark of color $\alpha=(r, g, b)$ and flavor $f_{a}=(u, d, s)$, and $\varepsilon_{i j k}$ denotes the Levi-Civita symbol. The latter is zero for $i=j, j=k$, or $k=i ;+1$ for $(i, j, k)$ an even permutation of $(1,2,3)$; and -1 for $(i, j, k)$ an odd permutation of $(1,2,3)$. The condensate is a Lorentz scalar, antisymmetric in Dirac indices, antisymmetric in color, and thus antisymmetric in flavor. The gap parameters $\Delta_{1}, \Delta_{2}$ and $\Delta_{3}$ describe $d-s, u-s$ and $u-d$ quark Cooper pairs, respectively. The following pairing schemes have emerged. At asymptotic densities $\left(m_{s} \rightarrow 0\right.$ or $\left.\mu \rightarrow \infty\right)$ the ground state of QCD with a vanishing strange quark mass is the color-flavor locked (CFL) phase (color-flavor locked quark pairing), in which all three quark flavors participate symmetrically. The gaps associated with this phase are

$$
\Delta_{3} \simeq \Delta_{2}=\Delta_{1}=\Delta,
$$

and the quark condensates of the CFL phase are approximately of the form

$$
\left\langle\psi_{f_{a}}^{\alpha} C \gamma_{5} \psi_{f_{b}}^{\beta}\right\rangle \sim \Delta \varepsilon^{\alpha \beta X} \varepsilon_{f_{a} f_{b} X},
$$

with color and flavor indices all running from 1 to 3 . Since $\varepsilon^{\alpha \beta X} \varepsilon_{f_{a} f_{b} X}=\delta_{f_{a}}^{\alpha} \delta_{f_{b}}^{\beta}-\delta_{f_{b}}^{\alpha} \delta_{f_{a}}^{\beta}$ one sees that the condensate (2.17) involves Kronecker delta functions that link color and flavor indices. Hence the notion color-flavor locking. The CFL phase has been shown to be electrically neutral without any need for electrons for a significant range of chemical potentials and strange quark masses [41]. If the strange quark mass is heavy enough to be ignored, then up and down quarks may pair in the two-flavor superconducting (2SC) phase. Other possible condensation patterns are CFL- $K^{0}$ [42], CFL- $K^{+}$and CFL- $\pi^{0,-}$ [43], gCFL (gapless CFL phase) [40], 1SC (singleflavor-pairing) [40, 44, 45], CSL (color-spin locked phase) [46], and the LOFF (crystalline pairing) [47, 48, 49] phase, depending on $m_{s}, \mu$, and electric charge density. Calculations performed for massless up and down quarks and a very heavy strange quark mass $\left(m_{s} \rightarrow \infty\right)$ agree that the quarks prefer to pair in the two-flavor superconducting (2SC) phase where

$$
\Delta_{3}>0, \quad \text { and } \quad \Delta_{2}=\Delta_{1}=0 .
$$

In this case the pairing ansatz (2.15) reduces to

$$
\left\langle\psi_{f_{a}}^{\alpha} C \gamma_{5} \psi_{f_{b}}^{\beta}\right\rangle \propto \Delta \varepsilon_{a b} \varepsilon^{\alpha \beta 3} .
$$


Here the resulting condensate picks a color direction ( 3 or blue in the example (2.19) above), and creates a gap $\Delta$ at the Fermi surfaces of quarks with the other two out of three colors (red and green). The gapless CFL phase (gCFL) may prevail over the CFL and 2SC phases at intermediate values of $m_{s}^{2} / \mu$ with gaps given obeying the relation

$$
\Delta_{3}>\Delta_{2}>\Delta_{1}>0
$$

For chemical potentials that are of astrophysical interest, $\mu<1000 \mathrm{MeV}$, the gap is between 50 and $100 \mathrm{MeV}$. The order of magnitude of this result agrees with calculations based on phenomenological effective interactions [39, 50] as well as with perturbative calculations for $\mu>10 \mathrm{GeV}$ [51]. We also note that superconductivity modifies the equation of state at the order of $(\Delta / \mu)^{2}[12,52]$, which is even for such large gaps only a few percent of the bulk energy. Such small effects may be safely neglected in present determinations of models for the equation of state of quark-hybrid stars. There has been much recent work on how color superconductivity in neutron stars could affect their properties (see Refs. [5, 6, 47, 53, 54, 55] and references therein). These studies reveal that possible signatures include the cooling by neutrino emission, the pattern of the arrival times of supernova neutrinos, the evolution of neutron star magnetic fields, rotational stellar instabilities, and glitches in rotation frequencies.

Aside from neutron star properties, an additional test of color superconductivity may be provided by upcoming cosmic ray space experiments such as AMS [56] and ECCO [57]. ${ }^{1}$ As shown in Ref. [58], finite lumps of color-flavor locked strange quark matter (see Sect. 2.6), which should be present in cosmic rays if strange matter is the ground state of the strong interaction, turn out to be significantly more stable than strangelets without color-flavor locking for wide ranges of parameters. In addition, strangelets made of CFL strange matter obey a charge-mass relation of $Z / A \propto A^{-1 / 3}$, which differs significantly from the charge-mass relation of strangelets made of ordinary strange quark matter. In the latter case, $Z / A$ would be constant for small baryon numbers $A$ and $Z / A \propto A^{-2 / 3}$ for large $A[10,58,59]$. This difference may allow an experimental test of CFL locking in strange quark matter [58].

\subsection{Absolute stability of strange quark matter}

So far we have assumed that quark matter forms a state of matter higher in energy than atomic nuclei. This most plausible assumption, however, may not be correct $[60,61,62]$, since for a collection of more than a few hundred $u, d, s$ quarks, the energy per baryon, $E / A$, of quark matter can be just as well below the energy per baryon of the most stable atomic nuclei, nickel and iron. This is known as the strange quark matter hypothesis. The energy per baryon in ${ }^{56} \mathrm{Fe}$, for instance, is given by $M\left({ }^{56} \mathrm{Fe}\right) c^{2} / 56=930.4 \mathrm{MeV}$, with $M\left({ }^{56} \mathrm{Fe}\right)$ the mass of the ${ }^{56} \mathrm{Fe}$ atom. A simple estimate shows that for strange quark matter described by the MIT bag model $E / A=4 B \pi^{2} / \mu^{3}$, so that bag constants of $B=57 \mathrm{MeV} / \mathrm{fm}^{3}$ (i.e. $B^{1 / 4}=145 \mathrm{MeV}$ ) and $B=85 \mathrm{MeV} / \mathrm{fm}^{3}\left(B^{1 / 4}=\right.$ $160 \mathrm{MeV}$ ) would place the energy per baryon of strange quark matter at $E / A=829 \mathrm{MeV}$ and $915 \mathrm{MeV}$, respectively, which correspond to strange quark matter which is absolutely bound with respect to nuclear matter [10]. If this were indeed the case, neutron star matter would be metastable with respect to strange quark matter, and all neutron stars should in fact be strange quark stars

\footnotetext{
${ }^{1}$ See J. Madsen's contribution on strange matter in cosmic rays published elsewhere in this volume.
} 


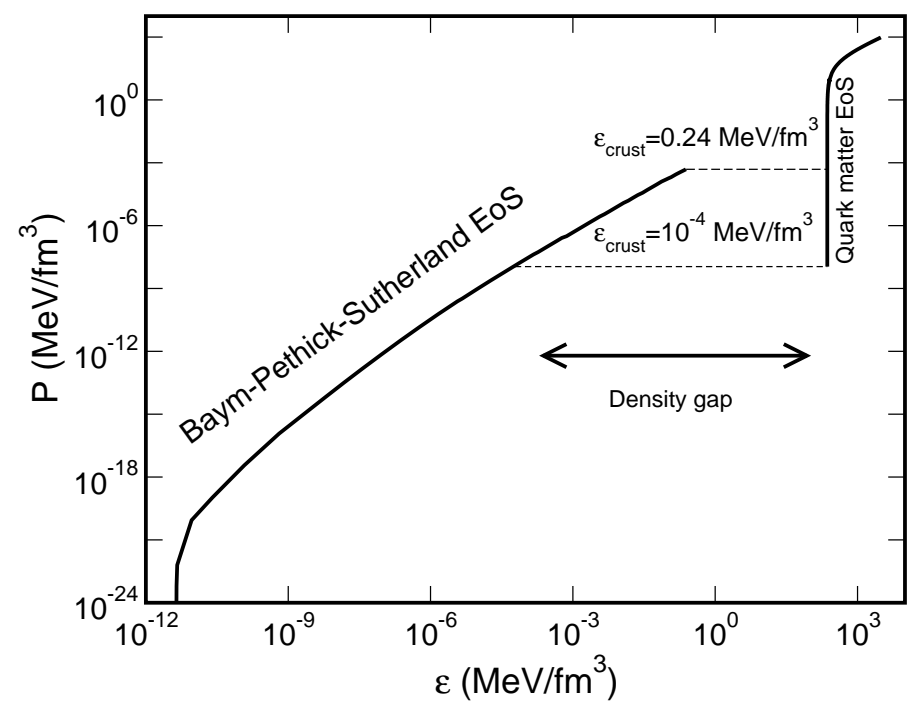

Figure 5: EoS of strange quark matter surrounded by nuclear matter. The maximal possible nuclear matter density is determined by neutron drip which occurs at $\varepsilon_{\text {crust }}=0.24 \mathrm{MeV} / \mathrm{fm}^{3}\left(4.3 \times 10^{11} \mathrm{~g} / \mathrm{cm}^{3}\right)$. Any nuclear density that is smaller than that is possible. As an example, we show here the EoS for a chosen density of $\varepsilon_{\text {crust }}=10^{-4} \mathrm{MeV} / \mathrm{fm}^{3}\left(10^{8} \mathrm{~g} / \mathrm{cm}^{3}\right)$.

$[10,63,64]$. As outlined just above, strange quark matter is expected to be a color superconductor which, at extremely high densities, should be in the CFL phase. This phase is rigorously electrically neutral with no electrons required [41]. For sufficiently large strange quark masses, however, the low density regime of strange quark matter is rather expected to form a 2SC phase (or possibly other phases) in which electrons are present $[5,6]$. The presence of electrons causes the formation of an electric dipole layer on the surface of strange matter, which enables strange quark matter stars to carry crusts made of ordinary nuclear matter $[8,9,65]$. The maximal possible density at the base of the crust (inner crust density) is determined by neutron drip, which occurs at about $4 \times 10^{11} \mathrm{~g} / \mathrm{cm}^{3}$. This somewhat complicated situation of the structure of strange matter enveloped in a (chemically equilibrated) nuclear crust can be represented by a proper choice for the EoS which consists of two parts [66]. At densities below neutron drip it can be represented by the EoS of Baym-PethickSutherland, while the high-density part, consisting of strange quark matter, can be described by the bag model EoS (see Fig. 5). The EoS is characterized by a significant discontinuity in density between strange quark matter and nuclear crust matter across the electric dipole gap where the pressure of the nuclear crust at its base equals the pressure of strange matter at its surface $[2,4,66]$.

One crucial astrophysical test of the strange quark matter hypothesis is whether strange quark stars can give rise to the observed phenomena of pulsar glitches. In the crust quake model an oblate solid nuclear crust in its present shape slowly comes out of equilibrium with the forces acting on it as the rotational period changes, and fractures when the built up stress exceeds the sheer strength of the crust material. The period and rate of change of period slowly heal to the trend preceding the glitch as the coupling between crust and core re-establish their co-rotation. The existence of glitches may have a decisive impact on the question of whether the strange quark matter hypothesis holds or not. From the calculations in [66] it is known that the ratio of the crustal moment of inertia 
to the star's total moment of inertia, $I_{\text {crust }} / I_{\text {total }}$, varies between $10^{-3}$ and $10^{-5}$ at the maximum mass. If the angular momentum of the pulsar is conserved in the quake then the relative frequency change and moment of inertia change are equal and one arrives at [66]

$$
\frac{\Delta \Omega}{\Omega}=\frac{|\Delta I|}{I_{0}}>\frac{|\Delta I|}{I} \equiv f \frac{I_{\text {crust }}}{I} \sim\left(10^{-5}-10^{-3}\right) f, \text { with } \quad 0<f<1 .
$$

Here $I_{0}$ denotes the moment of inertia of that part of the star whose frequency is changed in the quake. It might be that of the crust only, some fraction, or all of the star. The factor $f$ in Eq. (2.21) represents the fraction of the crustal moment of inertia that is altered in the quake, i.e., $f \equiv$ $|\Delta I| / I_{\text {crust }}$. Since the observed glitches have relative frequency changes $\Delta \Omega / \Omega=\left(10^{-9}-10^{-6}\right)$, a change in the crustal moment of inertia of $f \lesssim 0.1$ would cause a giant glitch even in the least favorable case [66]. Moreover, one finds that the observed range of the fractional change in the spin-down rate, $\dot{\Omega}$, is consistent with the crust having the small moment of inertia calculated and the quake involving only a small fraction $f$ of that, just as in Eq. (2.21). To this aim we write [66]

$$
\frac{\Delta \dot{\Omega}}{\dot{\Omega}}=\frac{\Delta \dot{\Omega} / \dot{\Omega}}{\Delta \Omega / \Omega} \frac{|\Delta I|}{I_{0}}=\frac{\Delta \dot{\Omega} / \dot{\Omega}}{\Delta \Omega / \Omega} f \frac{I_{\text {crust }}}{I_{0}}>\left(10^{-1} \text { to } 10\right) f,
$$

where use of Eq. (2.21) has been made. Equation (2.22) yields a small $f$ value in the range $f<$ $\left(10^{-4}\right.$ to $\left.10^{-1}\right)$, in agreement with $f \lesssim 10^{-1}$ established just above. Here measured values of the ratio $(\Delta \Omega / \Omega) /(\Delta \dot{\Omega} / \dot{\Omega}) \sim 10^{-6}$ to $10^{-4}$ for the Crab and Vela pulsars, respectively, have been used.

An improved discussion of the surface gap below strange star crusts has been performed very recently in Ref. [67]. In addition to the electrostatic forces described above, this study includes gravity too. The properties of the gap are investigated for a wide range of parameters assuming both color-flavor locked and noncolor-flavor locked strange star cores. It is found that the maximally allowed inner crust density is generally lower than that of neutron drip. This does not alter the overall form of the EoS shown in Fig. 5, however. Another interesting finding concerning the surface properties of strange stars was recently published in Ref. [68]. In this paper it is found that, depending on the surface tension of nuggets of strange matter, a heterogeneous crust comprised of nuggets of strange quark matter embedded in an uniform electron background may exist in the surface region of strange stars. This heterogeneous strange star surface would have a negligible electric field which would make the existence of an ordinary nuclear crust, which requires a very strong electric field, impossible.

\section{Models of compact stars}

Neutron stars are objects of highly compressed matter so that the geometry of space-time is changed considerably from flat space. Thus models of such stars are to be constructed in the framework of Einstein's general theory of relativity combined with theories of superdense matter. The effects of curved space-time are included by coupling the energy-momentum density tensor for matter fields to Einstein's field equations. The generally covariant Lagrangian density is

$$
\mathscr{L}=\mathscr{L}_{\mathrm{E}}+\mathscr{L}_{\mathrm{G}},
$$


where the dynamics of particles is introduced through the matter Lagrangian $\mathscr{L}_{\mathrm{M}}$ added to the gravitational Lagrangian $\mathscr{L}_{\mathrm{G}}$. The latter is given by

$$
\mathscr{L}_{\mathrm{G}}=g^{1 / 2} R=g^{1 / 2} g^{\mu v} R_{\mu v}
$$

where $g^{\mu v}$ and $R_{\mu v}$ denote the metric tensor and the Ricci tensor, respectively. The latter is given by

$$
R_{\mu v}=\Gamma_{\mu \sigma, v}^{\sigma}-\Gamma_{\mu \nu, \sigma}^{\sigma}+\Gamma_{\kappa \nu}^{\sigma} \Gamma_{\mu \sigma}^{\kappa}-\Gamma_{\kappa \sigma}^{\sigma} \Gamma_{\mu \nu}^{\kappa}
$$

where commas followed by a Greek letter denote derivatives with respect to space-time coordinates, e.g. , ${ }_{v}=\partial / \partial x^{v}$ etc. The Christoffel symbols $\Gamma$ in (3.3) are defined as

$$
\Gamma_{\mu \nu}^{\sigma}=\frac{1}{2} g^{\sigma \lambda}\left(g_{\mu \lambda, v}+g_{v \lambda, \mu}-g_{\mu v, \lambda}\right) .
$$

The connection between both branches of physics is provided by Einstein's field equations

$$
G^{\mu v} \equiv R^{\mu v}-\frac{1}{2} g^{\mu v} R=8 \pi T^{\mu v}(\varepsilon, P(\varepsilon)),
$$

$(\mu, v=0,1,2,3)$ which couples the Einstein curvature tensor, $G^{\mu v}$, to the energy-momentum density tensor, $T^{\mu v}$, of the stellar matter. The quantities $g^{\mu v}$ and $R$ in (3.5) denote the metric tensor and the Ricci scalar (scalar curvature) [2]. The tensor $T^{\mu v}$ contains the equation of state, $P(\varepsilon)$, of the stellar matter discussed in Sect. 2. In general, Einstein's field equations and the many-body equations were to be solved simultaneously since the baryons and quarks move in curved spacetime whose geometry, determined by Einstein's field equations, is coupled to the total mass energy density of the matter. In the case of neutron stars, as for all astrophysical situations for which the long-range gravitational forces can be cleanly separated from the short-range forces, the deviation from flat space-time over the length scale of the strong interaction, $\sim 1 \mathrm{fm}$, is however practically zero up to the highest densities reached in the cores of such stars (some $10^{15} \mathrm{~g} / \mathrm{cm}^{3}$ ). This is not to be confused with the global length scale of neutron stars, $\sim 10 \mathrm{~km}$, for which $M / R \sim 0.3$, depending on the star's mass. That is to say, gravity curves space-time only on a macroscopic length scale but leaves it flat to a very good approximation on a microscopic length scale. To achieve an appreciable curvature on a microscopic scale set by the strong interaction, mass densities greater than $\sim 10^{40} \mathrm{~g} / \mathrm{cm}^{3}$ would be necessary [69]! This circumstance divides the construction of models of compact stars into two distinct problems. Firstly, the effects of the short-range nuclear forces on the properties of matter are described in a comoving proper reference frame (local inertial frame), where space-time is flat, by the parameters and laws of special relativistic many-body physics. Secondly, the coupling between the long-range gravitational field and the matter is then taken into account by solving Einstein's field equations for the gravitational field described by the general relativistic curvature of space-time, which determines the global structure of the stellar configuration.

For many studies of neutron star properties it is sufficient to treat neutron star matter as a perfect fluid. The energy-momentum tensor of such a fluid is given by

$$
T^{\mu v}=\frac{d x^{\mu}}{d \tau} \frac{d x^{v}}{d \tau}(\varepsilon+P)+g^{\mu v} P .
$$



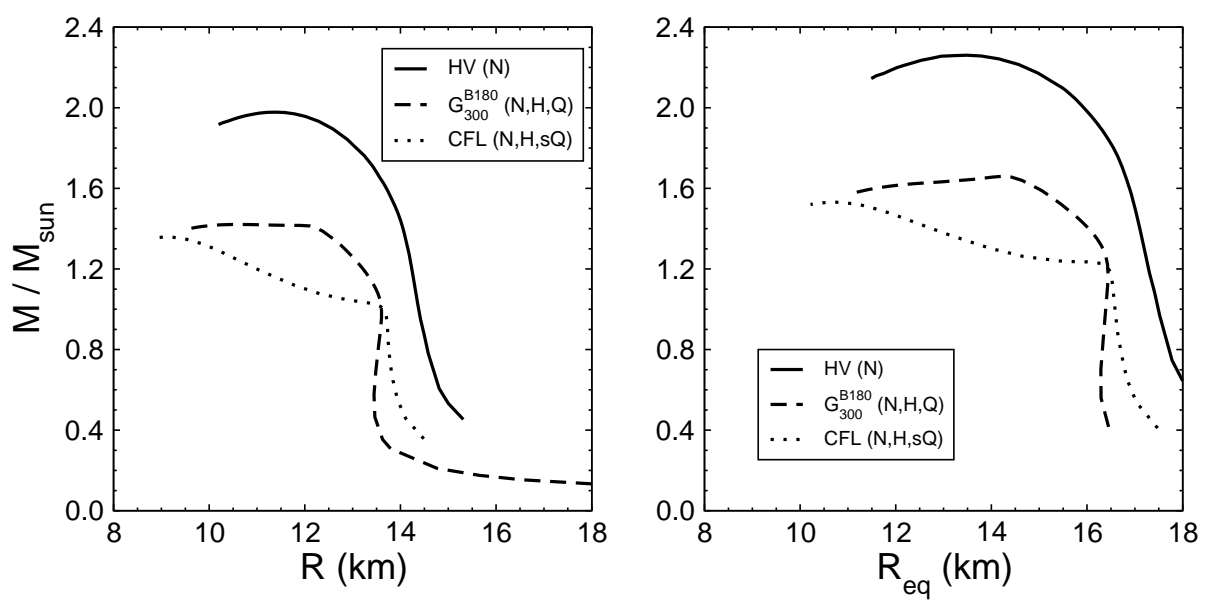

Figure 6: Mass-radius relations of non-rotating (left panel) and rotating (right panel: $\Omega=\Omega_{K}$ ) neutron stars computed for different EoSs. $\Omega_{K}$ denotes the general relativistic Kepler (mass shedding) frequency.

For non-rotating spherically symmetric stars the metric has the rather simple form

$$
d s^{2}=-e^{2 \Phi(r)} d t^{2}+e^{2 \Lambda(r)} d r^{2}+r^{2} d \theta^{2}+r^{2} \sin ^{2} \theta d \phi^{2},
$$

where $\Phi(r)$ and $\Lambda(r)$ are radially varying metric functions. From (3.7) one reads off the following covariant components of the metric tensor,

$$
g_{t t}=-e^{2 \Phi(r)}, g_{r r}=e^{2 \Lambda(r)}, g_{\theta \theta}=r^{2}, g_{\phi \phi}=r^{2} \sin ^{2} \theta
$$

so that the only non-vanishing Christoffel symbols are

$$
\begin{aligned}
& \Gamma_{t t}^{r}=e^{2 \Phi(r)-2 \Lambda(r)} \Phi^{\prime}(r), \Gamma_{t r}^{t}=\Phi^{\prime}(r), \Gamma_{r r}^{r}=\Lambda^{\prime}(r), \Gamma_{r \theta}^{\theta}=r^{-1}, \Gamma_{r \phi}^{\phi}=r^{-1}, \Gamma_{\theta \theta}^{r}=-r e^{-2 \Lambda(r),} \\
& \Gamma_{\theta \phi}^{\phi}=\frac{\cos \theta}{\sin \theta}, \Gamma_{\phi \phi}^{r}=-r \sin ^{2} \theta e^{-2 \Lambda(r)}, \Gamma_{\phi \phi}^{\theta}=-\sin \theta \cos \theta,
\end{aligned}
$$

where primes denote differentiation with respect to the radial coordinate. Substituting (3.6) and (3.9) into Einstein's field equations leads to the general relativistic equations of hydrostatic equilibrium discussed first by Tolman [70] and Oppenheimer-Volkoff [71],

$$
\frac{d P(r)}{d r}=-\frac{\varepsilon(r) m(r)}{r^{2}} \frac{\left(1+\frac{P(r)}{\varepsilon(r)}\right)\left(1+\frac{4 \pi r^{3} P(r)}{m(r)}\right)}{1-\frac{2 m(r)}{r}} .
$$

Note that we use geometrized units, where the gravitational constant and velocity of light are $G=c=1$ so that $M_{\odot}=1.475 \mathrm{~km}$. The boundary condition to (3.10) is $P(r=0)=P\left(\varepsilon_{c}\right)$, where $\varepsilon_{c}$ denotes the energy density at the star's center, which constitutes a parameter. Equation (3.10) is to be integrated out to a radial distance where $P(r)=0$ which determines the star's radius, $R$. The mass contained in a sphere of radius $r(\leq R)$, denoted by $m(r)$, follows from $m(r)=4 \pi \int_{0}^{r} d r^{\prime} r^{\prime 2} \varepsilon\left(r^{\prime}\right)$. The star's total gravitational mass is thus given by $M \equiv m(R)$. Figure 6 shows the mass-radius relationships of both non-rotating as well as rotating sequences of neutron stars for the sample 
EoSs discussed in Sect. 2. The non-rotating sequences are solutions of the Tolman-OppenheimerVolkoff equation shown in (3.10). The construction of rotating sequences will be discussed shortly below. Figure 7 shows the gravitational mass of non-rotating as well as rotating neutron stars as a function of central star density. Stars to the right of the respective mass peaks in each panel are unstable against radial oscillations and thus cannot exist stably in nature. Also shown in these plots are the evolutionary (constant stellar baryon number, $A$ ) paths that isolated rotating neutron stars would follow during their stellar spin-down evolution caused by the emission of magnetic dipole radiation and a wind of $e^{+}-e^{-}$pairs. Figure 7 reveals that CFL stars may spend considerably more time in the spin-down phase than their competitors of the same mass. Another point that we want to
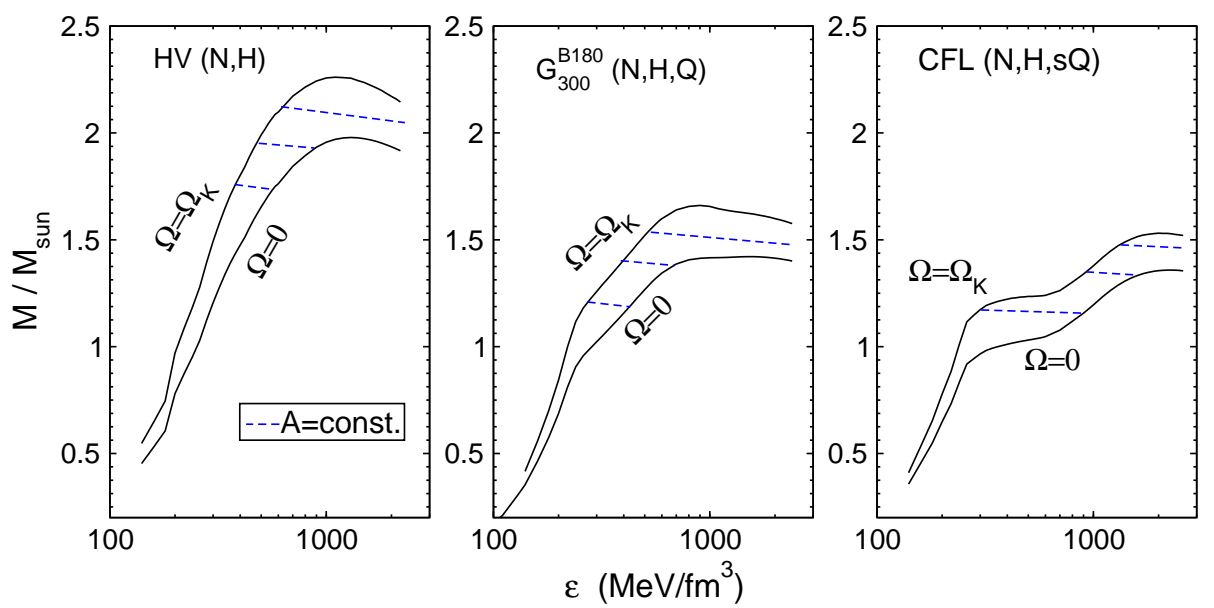

Figure 7: Mass-central energy relations for the sample equations of state introduced in Sect. 2.

make is that all equations of state are able to support neutron stars of canonical mass, $M \sim 1.4 M_{\odot}$. Neutron stars more massive than about $2 M_{\odot}$, on the other hand, are only supported by equations of state that exhibit a very stiff behavior at asymptotic densities, disfavoring the presence of hyperons, meson condensates, or quarks. Knowledge of the maximum possible mass of neutron stars is of great importance for two reasons. Firstly, because the largest known neutron star mass imposes a lower bound on the maximum mass of a theoretical model. Very massive neutron star candidates are J0751+1807 $\left(2.1_{-0.5}^{+0.4} M_{\odot}[72]\right)$, Vela X-1 $\left(1.88 \pm 0.13 M_{\odot}\right.$ if the inclination angle of the system is $i=90^{\circ}$; an inclination angle of $i=70^{\circ}$ increases the star's mass to $2.27 \pm 0.17 M_{\odot}$ [73]), and Cyg X-2 (1.78 $\pm 0.23 M_{\odot}$ [74]. Titarchuck and Shaposhnikov obtain for Cyg X-2 a lower mass of $\left.1.44 \pm 0.06 M_{\odot}[75]\right)$. The second reason is that the maximum mass of neutron stars is essential in order to identify solar-mass black hole candidates [76, 77].

The structure equations of rotating compact stars are considerably more complicated that those of non-rotating compact stars [2]. These complications have their cause in the rotational deformation, that is, a flattening at the pole accompanied with a radial blowup in the equatorial direction, which leads to a dependence of the star's metric on the polar coordinate, $\theta$, in addition to the mere dependence on the radial coordinate, $r$. Secondly, rotation stabilizes a star against gravitational collapse. A rotating star can therefore carry more mass than a non-rotating star. Being more massive, however, means that the geometry of space-time is changed too. This makes the 


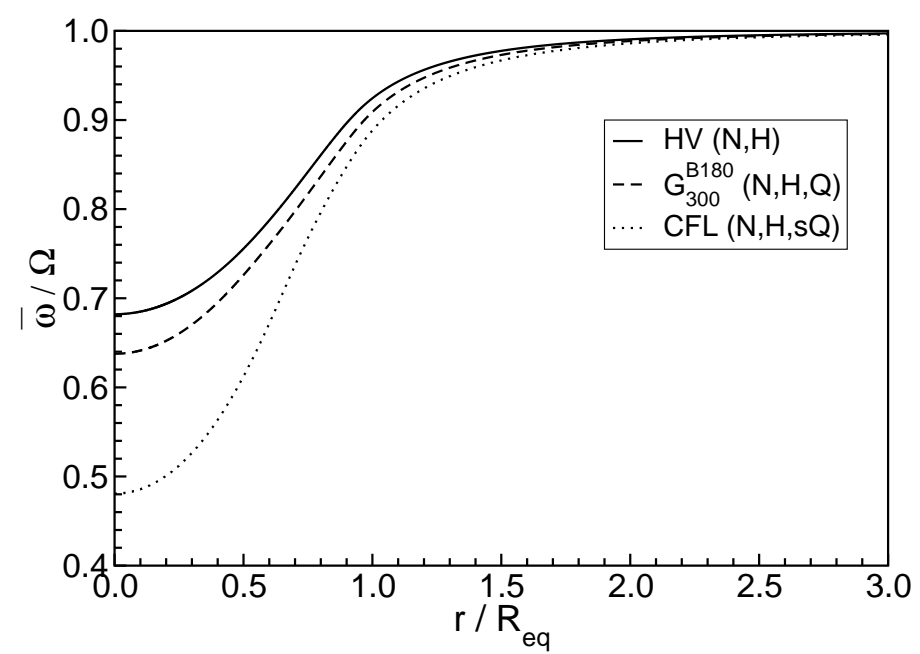

Figure 8: Dragging of local inertial frames (Lense-Thirring effect) caused by $\sim 1.4 M_{\odot}$ neutron stars rotating at $2 \mathrm{~ms}$. The frequency $\bar{\omega}$ is defined in Eq. (3.13).

metric functions associated with a rotating star depend on the star's rotational frequency. Finally, the general relativistic effect of the dragging of local inertial frames implies the occurrence of an additional non-diagonal term, $g^{t \phi}$, in the metric tensor $g^{\mu \nu}$. This term imposes a self-consistency condition on the stellar structure equations, since the extent to which the local inertial frames are dragged along by the star is determined by the initially unknown stellar properties like mass and limiting rotational frequency. The covariant components of the metric tensor of a rotating compact star are thus given by $[2,78]$

$$
g_{t t}=-e^{2 v}+e^{2 \psi} \omega^{2}, g_{t \phi}=-e^{2 \psi} \omega, g_{r r}=e^{2 \lambda}, g_{\theta \theta}=e^{2 \mu}, g_{\phi \phi}=e^{2 \psi},
$$

which leads for the line element to

$$
d s^{2}=g_{\mu v} d x^{\mu} d x^{v}=-e^{2 v} d t^{2}+e^{2 \psi}(d \phi-\omega d t)^{2}+e^{2 \mu} d \theta^{2}+e^{2 \lambda} d r^{2} .
$$

Here each metric function, i.e. $v, \psi, \mu$ and $\lambda$, as well as the angular velocities of the local inertial frames, $\omega$, depend on the radial coordinate $r$ and polar angle $\theta$ and implicitly on the star's angular velocity $\Omega$. Of particular interest is the relative angular frame dragging frequency, $\bar{\omega}$, defined as

$$
\bar{\omega}(r, \theta, \Omega) \equiv \Omega-\omega(r, \theta, \Omega)
$$

which is the angular velocity of the star, $\Omega$, relative to the angular velocity of a local inertial frame, $\omega$. It is this frequency that is of relevance when discussing the rotational flow of the fluid inside the star, since the magnitude of the centrifugal force acting on a fluid element is governed-in general relativity as well as in Newtonian gravitational theory-by the rate of rotation of the fluid element relative to a local inertial frame [79]. In contrast to Newtonian theory, however, the inertial frames inside (and outside) a general relativistic fluid are not at rest with respect to the distant stars, as pointed out just above. Rather, the local inertial frames are dragged along by the rotating fluid. Depending on the internal stellar constitution, this effect can be quite strong, as shown in Fig. 8 for 
Table 1: Properties of neutron stars composed of nucleons and hyperons (HV), nucleons, hyperons, and normal quarks $\left(\mathrm{G}_{300}^{\mathrm{B} 180}\right)$, and nucleons, hyperons, and color-superconducting quarks (CFL).

\begin{tabular}{|c|c|c|c|c|c|c|}
\hline & $\mathrm{HV}$ & $\mathrm{G}_{300}^{\mathrm{B} 180}$ & $\mathrm{CFL}$ & $\mathrm{HV}$ & $\mathrm{G}_{300}^{\mathrm{B} 180}$ & $\mathrm{CFL}$ \\
& $v=0$ & $v=0$ & $v_{\mathrm{K}}=0$ & $v_{\mathrm{K}}=850 \mathrm{~Hz}$ & $v_{\mathrm{K}}=940 \mathrm{~Hz}$ & $v_{\mathrm{K}}=1400 \mathrm{~Hz}$ \\
\hline \hline$\varepsilon_{\mathrm{c}}\left(\mathrm{MeV} / \mathrm{fm}^{3}\right)$ & 361.0 & 814.3 & 2300.0 & 280.0 & 400.0 & 1100.0 \\
\hline$I\left(\mathrm{~km}^{3}\right)$ & 0 & 0 & 0 & 223.6 & 217.1 & 131.8 \\
\hline$M\left(M_{\odot}\right)$ & 1.39 & 1.40 & 1.36 & 1.39 & 1.40 & 1.41 \\
\hline$R(\mathrm{~km})$ & 14.1 & 12.2 & 9.0 & 17.1 & 16.0 & 12.6 \\
\hline$Z_{\mathrm{p}}$ & 0.1889 & 0.2322 & 0.3356 & 0.2374 & 0.2646 & 0.3618 \\
$Z_{\mathrm{F}}$ & 0.1889 & 0.2322 & 0.3356 & -0.1788 & -0.1817 & -0.2184 \\
$Z_{\mathrm{B}}$ & 0.1889 & 0.2322 & 0.3356 & 0.6046 & 0.6502 & 0.9190 \\
\hline$g_{\mathrm{s}, 14\left(\mathrm{~cm} / \mathrm{s}^{2}\right)}$ & 1.1086 & 1.5447 & 3.0146 & 0.7278 & 0.8487 & 1.4493 \\
\hline$T / W$ & 0 & 0 & 0 & 0.0894 & 0.0941 & 0.0787 \\
\hline$B E\left(M_{\odot}\right)$ & 0.0937 & 0.1470 & 0.1534 & 0.0524 & 0.1097 & 0.1203 \\
\hline$V_{\mathrm{eq}} / c$ & 0 & 0 & 0 & 0.336 & 0.353 & 0.424 \\
\hline
\end{tabular}

rotating $2 \mathrm{~ms}$ neutron stars. For a very compact neutron star containing a color-superconducting CFL core, as in our example, one reads off from this figure that the local inertial frames at the star's center rotate at about half the star's rotational frequency, $\omega(r=0) \simeq \Omega / 2$. This value drops to about $15 \%$ for the local inertial frames located at the star's equator. The scenarios shown in Fig. 8 may be of great importance for binary millisecond neutron stars in their final accretion stages, where the accretion disk approaches the star very closely.

Table 1 summarizes the impact of strangeness on several intriguing properties of non-rotating as well as rotating neutron stars. The latter spin at their respective Kepler frequencies. One sees that the central energy density, $\varepsilon_{\mathrm{c}}$, spans a very wide range, depending on particle composition. The surface redshift is of importance since it is connected to observed neutron star temperatures through the relation $T^{\infty} / T_{\text {eff }}=1 /(1+Z)$. CFL quark stars may have redshifts that are up to $50 \%$ higher than those of conventional stars. Finally, we also show in Table 1 the surface gravity of stars, $g_{\mathrm{s}, 14}$ [80], which again may be up to $50 \%$ higher for CFL stars. The other quantities listed are the rotational kinetic energy in units of the total energy of the star, $T / W$, the stellar binding energy, $B E$, and the rotational velocity of a particle at the star's equator [2].

\section{Limiting rotational periods}

\subsection{Mass shedding from the equator}

No simple stability criteria are known for rapidly rotating stellar configurations in general relativity. However, an absolute limit on rapid rotation is set by the onset of mass shedding from the equator of a rotating star. The corresponding rotational frequency is known as the Kepler frequency, $\Omega_{\mathrm{K}}$. In classical mechanics, the expression for the Kepler frequency, determined by the equality between the centrifugal force and gravity, is readily obtained as $\Omega_{\mathrm{K}}=\sqrt{M / R^{3}}$. In order 


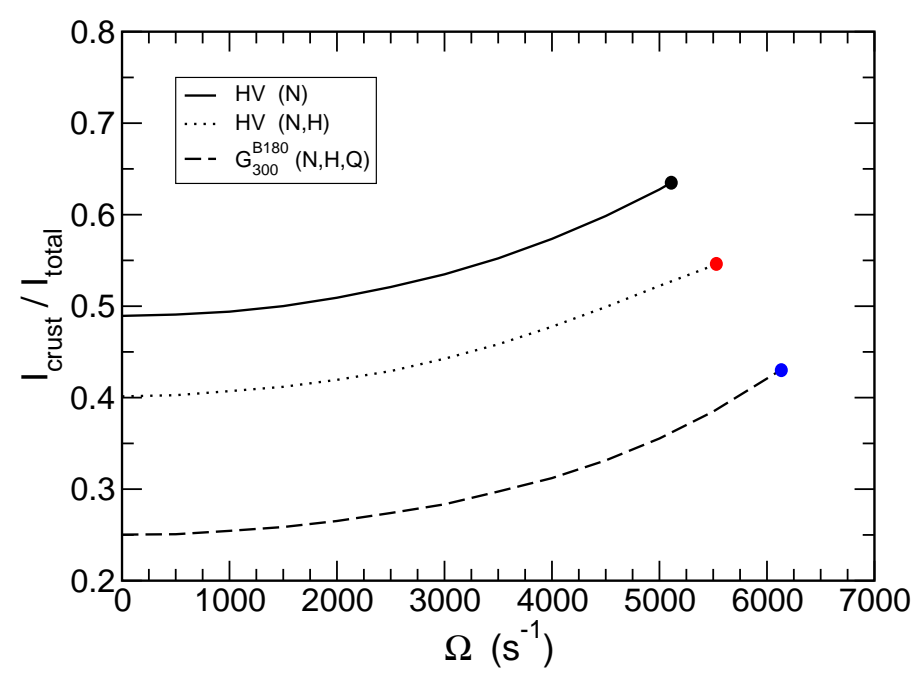

Figure 9: Moment of inertia of several sample neutron stars.

to derive the general relativistic counterpart of this relation, one applies the extremal principle to the circular orbit of a point mass rotating at the star's equator. Since $r=\theta=$ const for a point mass there, one has $d r=d \theta=0$. The line element (3.12) then reduces to

$$
d s^{2}=\left(e^{2 v}-e^{2 \psi}(\Omega-\omega)^{2}\right) d t^{2} .
$$

Substituting this expression into $J \equiv \int_{s_{1}}^{s_{2}} d s$, where $s_{1}$ and $s_{2}$ refer to points located at that particular orbit for which $J$ becomes extremal, gives

$$
J=\int_{s_{1}}^{s_{2}} d t \sqrt{e^{2 v}-e^{2 \psi}(\Omega-\omega)^{2}} .
$$

Applying the extremal condition $\delta J=0$ to Eq. (4.2) and noticing that $V=e^{\psi-v}(\Omega-\omega)$ then leads to the following relation,

$$
\frac{\partial \psi}{\partial r} e^{2 v} V^{2}-\frac{\partial \omega}{\partial r} e^{v+\psi} V-\frac{\partial v}{\partial r} e^{2 v}=0 .
$$

It constitutes a simple quadratic equation for the orbital velocity $V$ of a particle at the star's equator. One thus obtains for the Kepler frequency $\Omega_{\mathrm{K}}$ (Kepler period, $P_{\mathrm{K}}$ ) the final relation [2],

$$
\Omega_{\mathrm{K}}=\omega+\frac{\omega^{\prime}}{2 \psi^{\prime}}+e^{v-\psi} \sqrt{\frac{v^{\prime}}{\psi^{\prime}}+\left(\frac{\omega^{\prime}}{2 \psi^{\prime}} e^{\psi-v}\right)^{2}} \Rightarrow P_{\mathrm{K}}=\frac{2 \pi}{\Omega_{\mathrm{K}}},
$$

which is to be determined self-consistently at the star's equator (primes denote radial derivatives). For most neutron star matter equations of state, the Kepler period obtained for $1.4 M_{\odot}$ neutron stars scatters around $1 \mathrm{~ms}$. One exception to this are strange quark matter stars. These are self-bound and, thus, tend to possess smaller radii than conventional neutron stars, which are bound by gravity only. Because of their smaller radii, strange stars can withstand mass shedding down to periods of around $0.5 \mathrm{~ms}[66,81]$. CFL stars reside between these limits. 

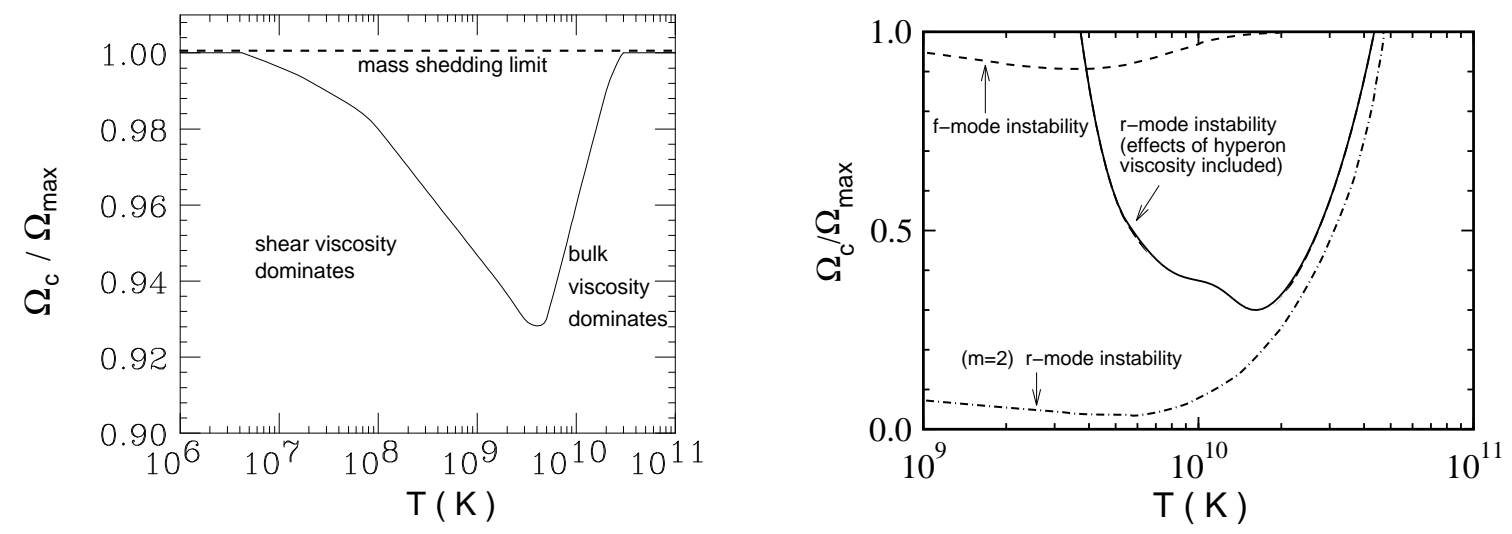

Figure 10: Temperature dependence of the critical angular velocity $\Omega_{\mathrm{c}}$ of rotating neutron stars. The left panel shows the gravitational radiation driven $f$-mode instability suppressed by shear and bulk viscosity. Right panel: comparison of $f$-mode instability with $r$-mode instability. (Data from Refs. [82, 83].)

As a last topic of this section, we briefly discuss the moment of inertia of a rotationally deformed star described by the metric in Eq. (3.12). For such stars the moment of inertia is given by

$$
I(\Omega)=2 \pi \int_{0}^{\pi} d \theta \int_{0}^{R(\theta)} d r e^{\lambda+\mu+v+\psi} \frac{\varepsilon+P(\varepsilon)}{e^{2 v-2 \psi}-(\omega-\Omega)^{2}} \frac{\Omega-\omega}{\Omega} .
$$

Figure 9 shows that the crustal fraction of the moment of inertia of a neutron star may be around $50 \%$ smaller if the star contains a very soft phase of matter like quark matter. This may be of relevance for pulsar glitch models and the modeling of the post-glitch behavior of pulsars.

\subsection{Gravitational radiation reaction driven instabilities}

Rotational instabilities in rotating stars, known as gravitational radiation driven instabilities, are probably setting a more stringent limit on rapid stellar rotation than mass shedding. These instabilities originate from counter-rotating surface vibrational modes which at sufficiently high rotational star frequencies are dragged forward. In this case gravitational radiation, which inevitably accompanies the aspherical transport of matter, does not damp the instability modes but rather drives them. Viscosity plays the important role of damping these instabilities at a sufficiently reduced rotational frequency such that the viscous damping rate and power in gravity waves are comparable. The most critical instability modes that are driven unstable by gravitational radiation are $f$-modes and $r$-modes. Figure 10 shows the stable neutron star frequencies if only $f$-modes were operative. One sees that hot as well as cold neutron stars can rotate at frequencies close to mass shedding, because of the large contributions of shear and bulk viscosity, respectively, for this temperature regime. The more recently discovered $r$-mode instability may change the picture completely, as can be seen too from Fig. 10. These modes are driven unstable by gravitational radiation over a considerably wider range of angular velocities than the $f$-modes (cf. dashed curve labeled $(m=2) r$-mode instability). In stars with cores cooler than $\sim 10^{9} \mathrm{~K}$, on the other hand, the $r$-mode instability may be completely suppressed by viscous phenomena so that stable rotation would be limited by the $f$-mode instability again [82]. 


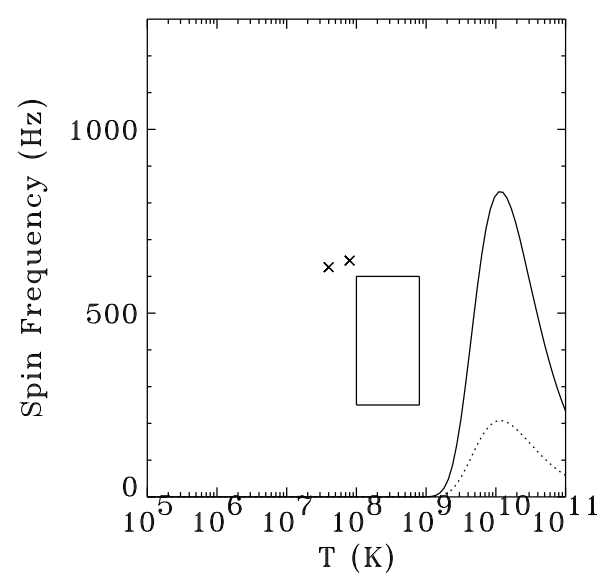

Figure 11: Critical rotation frequencies versus stellar temperature for CFL strange stars [85].

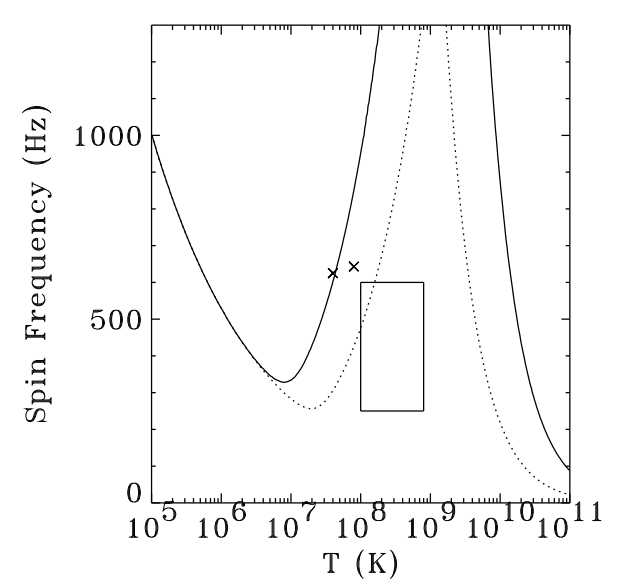

Figure 12: Same as Fig. 11, but for 2SC quark stars [85].

Figures 11 and 12 are the counterparts to Fig. 10 but calculated for strange stars made of CFL and 2SC quark matter, respectively $[84,85]$. The $r$-mode instability seems to rule out that pulsars are CFL strange stars, if the characteristic time scale for viscous damping of $r$-modes are exponentially increased by factors of $\sim \Delta / T$ as calculated in [84]. An energy gap as small as $\Delta=$ $1 \mathrm{MeV}$ was assumed. For much larger gaps of $\Delta \sim 100 \mathrm{MeV}$, as expected for color superconducting quark matter in the CFL phase, the entire diagram would be $r$-mode unstable. The full curve in Fig. 11 is calculated for a strange quark mass of $m_{s}=200 \mathrm{MeV}$, the dotted curve for $m_{s}=100 \mathrm{MeV}$. The box marks the positions of most low mass X-ray binaries (LMXBs) [86], and the crosses denote the most rapidly rotating millisecond pulsars known. All strange stars above the curves would spin down on a time scale of hours due to the $r$-mode instability, in complete contradiction to the observation of millisecond pulsars and LMXBs, which would rule out CFL quark matter in strange stars (see, however, Ref. [87]). Figure 12 shows the critical rotation frequencies of quark stars as a function of internal stellar temperature for $2 \mathrm{SC}$ quark stars. For such quark stars the situation is less conclusive. Rapid spin-down, driven by the $r$-mode gravitational radiation instability, would happen for stars above the curves.

\section{Astrophysical signals of quark deconfinement}

\subsection{Isolated, rotating neutron stars}

Whether or not quark deconfinement occurs in neutron stars makes only very little difference to their static properties, such as the range of possible masses and radii, which renders the detection of quark matter in such objects extremely complicated. This may be strikingly different for isolated, rotating neutron stars which spin down, and thus become more compressed, because of the emission of magnetic dipole radiation and a wind of electron-positron pairs. For some rotating neutron stars the mass and initial rotational frequency may be just such that the central density rises from below to above the critical density for dissolution of baryons into their quark constituents. If accompanied 
by a pronounced shrinkage of the neutron star, as is the case for the neutron star shown in the central panel in Fig. 4, the star's moment of inertia could change dramatically. As shown in [88], the moment of inertia can decrease so anomalously that it could even introduce an era stellar spinup that may last for $\sim 10^{8}$ years. Since the dipole age of millisecond pulsars is about $10^{9}$ years, one may roughly estimate that about $10 \%$ of the solitary millisecond pulsars could be in the quark transition epoch and thus could be signaling the ongoing process of quark deconfinement. Changes in the moment of inertia reflect themselves in the braking index, $n$, of a rotating neutron star, as can be seen from $[4,88,89]$

$$
n(\Omega) \equiv \frac{\Omega \ddot{\Omega}}{\dot{\Omega}^{2}}=3-\frac{I+3 I^{\prime} \Omega+I^{\prime \prime} \Omega^{2}}{I+I^{\prime} \Omega} \simeq 3-\frac{3 I^{\prime} \Omega+I^{\prime \prime} \Omega^{2}}{2 I+I^{\prime} \Omega}
$$

where dots (primes) denote derivatives with respect to time $(\Omega)$. The last relation in (5.1) constitutes the non-relativistic limit of the braking index [90]. It is obvious that these expressions reduce to the canonical limit $n=3$ if $I$ is independent of frequency. Evidently, this is not the case for rapidly

Table 2: Dominant neutrino emitting processes in neutron star cores if hyperons and quarks are absent [21].

\begin{tabular}{|c|c|c|c|}
\hline Name & Process & $\begin{array}{c}\text { Emissivity } \\
\left(\mathrm{erg} \mathrm{cm}^{-3} \mathrm{~s}^{-1}\right)\end{array}$ & \\
\hline $\begin{array}{l}\text { Modified Urca cycle } \\
\text { (neutron branch) }\end{array}$ & $\begin{array}{l}n+n \rightarrow n+p+e^{-}+\bar{v}_{e} \\
n+p+e^{-} \rightarrow n+n+v_{e}\end{array}$ & $\sim 2 \times 10^{21} R T_{9}^{8}$ & Slow \\
\hline $\begin{array}{l}\text { Modified Urca cycle } \\
\text { (proton branch) }\end{array}$ & $\begin{array}{l}p+n \rightarrow p+p+e^{-}+\bar{v}_{e} \\
p+p+e^{-} \stackrel{p+n+v_{e}}{\rightarrow}+n+v^{2}\end{array}$ & $\sim 10^{21} R T_{9}^{8}$ & Slow \\
\hline Bremsstrahlung & $\begin{array}{l}n+n \rightarrow n+n+v+\bar{v} \\
n+p \rightarrow n+p+v+\bar{v} \\
p+p \rightarrow p+p+v+\bar{v}\end{array}$ & $\sim 10^{19} R T_{9}^{8}$ & Slow \\
\hline $\begin{array}{l}\text { Cooper pair } \\
\text { formations }\end{array}$ & $\begin{array}{l}n+n \rightarrow[n n]+v+\bar{v} \\
p+p \rightarrow[p p]+v+\bar{v}\end{array}$ & $\begin{array}{l}\sim 5 \times 10^{21} R T_{9}^{7} \\
\sim 5 \times 10^{19} R T_{9}^{7}\end{array}$ & Medium \\
\hline Direct Urca cycle & $\mid \begin{array}{l}n \rightarrow p+e^{-}+\bar{v}_{e} \\
p+e^{-} \rightarrow n+v_{e}\end{array}$ & $\sim 10^{27} R T_{9}^{6}$ & Fast \\
\hline$\pi^{-}$condensate & $n+<\pi^{-}>\rightarrow n+e^{-}+\bar{v}_{e}$ & $\sim 10^{26} R T_{9}^{6}$ & Fast \\
\hline$K^{-}$condensate & $n+<K^{-}>\rightarrow n+e^{-}+\bar{v}_{e}$ & $\sim 10^{25} R T_{9}^{6}$ & Fast \\
\hline
\end{tabular}

rotating neutron stars, and it also fails for stars that undergo pronounced compositional changes (phase transitions) which alter the moment of inertia significantly. Under favorable circumstances, these changes in $I$, originating from the transition of confined hadronic matter into quark matter, may cause the braking index to deviate dramatically from 3 in the vicinity of the star's frequency where the phase transition to quark matter occurs. The changes in I may even be so pronounced that $n(\Omega) \rightarrow \pm \infty$ at the transition point $[1,2,4,90]$. Such dramatic anomalies in $n(\Omega)$ are not known for conventional neutron stars (see left panel in Fig. 4), because their radii and thus moments of inertia appear to vary smoothly with $\Omega$ [2, 4]. A counterexample to this, however, is discussed in [91]. The future astrophysical observation of strong anomalies in the braking behavior of isolated pulsars could thus be cautiously interpreted as a possible astrophysical signal for quark deconfinement in neutron stars. 
Table 3: Dominant neutrino emitting processes in deconfined quark matter [21].

\begin{tabular}{|llcc|}
\hline Name & Process & $\begin{array}{c}\text { Emissivity } \\
\left(\mathrm{erg} \mathrm{cm}^{-3} \mathrm{~s}^{-1}\right)\end{array}$ & Efficiency \\
\hline $\begin{array}{l}\text { Direct Urca cycle } \\
\text { (ud branch) }\end{array}$ & $\begin{array}{l}u+e^{-} \rightarrow d+\underline{v}_{e} \\
d \rightarrow u+e^{-}+\bar{v}_{e}\end{array}$ & $\sim 10^{26} R T_{9}^{6}$ & Fast \\
$\begin{array}{l}\text { Direct Urca cycle } \\
\text { (us branch) }\end{array}$ & $\begin{array}{l}u+e^{-} \rightarrow s+\underline{v}_{e} \\
s \rightarrow u+e^{-}+\bar{v}_{e}\end{array}$ & $\sim 10^{25} R T_{9}^{6}$ & Fast \\
$\begin{array}{l}\text { Modified Urca cycle } \\
\text { (ud branch) }\end{array}$ & $\begin{array}{l}Q+u+e^{-} \rightarrow Q+d+v_{e} \\
\text { Modified Urca cycle }\end{array}$ & $\sim 10^{21} R T_{9}^{8}$ & Slow \\
$\begin{array}{l}\text { (us branch) } \\
\text { Bremsstrahlungs }\end{array}$ & $\begin{array}{l}Q+u+e^{-} \rightarrow Q+s+e^{-}+\bar{v}_{e} \\
Q+s \rightarrow Q+u+e^{-} \bar{v}_{e}\end{array}$ & $\sim 10^{20} R T_{9}^{8}$ & Slow \\
$\begin{array}{l}\text { Cooper pair } \\
\text { formations }\end{array}$ & $\begin{array}{l}u+u \rightarrow[u u]+v+\bar{v} \\
d+d \rightarrow[d d]+v+\bar{v}\end{array}$ & $\sim 2.5 \times 10^{20} R T_{9}^{7}$ & Slow \\
\hline
\end{tabular}

\subsection{Accreting neutron stars}

Accreting x-ray neutron stars provide a very interesting contrast to the spin-down of isolated neutron stars. These x-ray neutron stars are being spun up by the accretion of matter from a lowermass $\left(M \lesssim 0.4 M_{\odot}\right)$, less-dense companion. If the critical deconfinement density falls within that of the canonical pulsars, quark matter could already exist in them but will be spun out of such stars as their frequency increases during accretion. This scenario has been modeled in [92], where it was found that quark matter remains relatively dormant in the core of a neutron star until the star has been spun up to frequencies at which the central density is about to drop below the threshold density at which quark matter exists. As known from the discussion above, this could manifest itself in a significant increase of the star's moment of inertia. The angular momentum added to a neutron star during this phase of evolution is therefore consumed by the star's expansion, inhibiting a further spin-up until the star's quark matter content has been completely converted into a mixed phase of hadrons and quarks. Such accreters, therefore, tend to spend a greater length of time in the critical frequencies than otherwise. For canonical accretion rates of $10^{-10} M_{\odot} /$ year the time span can be on the order of $10^{9}$ years. Hence, from this scenario, one would expect a greater number of accreting x-ray neutron stars that appear near the same frequency. Evidence that accreting neutron stars pile up at certain frequencies, which are well below the mass shedding limit, is provided by the spin distribution of accreting millisecond pulsars in 57 Tuc and neutron stars in low mass X-ray binaries observed with the Rossi X-ray Timing Explorer (RXTE). The proposed limiting mechanisms responsible for this behavior is generally attributed to gravity-wave emission caused by the $r$-mode instability, or by a small stellar mass quadrupole moment $[93,94,95]$. Supplemental to these explanations, quark reconfinement (or, more generally, strong first-order like phase transition) may be linked to this phenomenon as well $[37,92,96,97]$.

\section{Cooling of neutron stars}

The predominant cooling mechanism of hot (temperatures of several $\sim 10^{10} \mathrm{~K}$ ) newly formed 


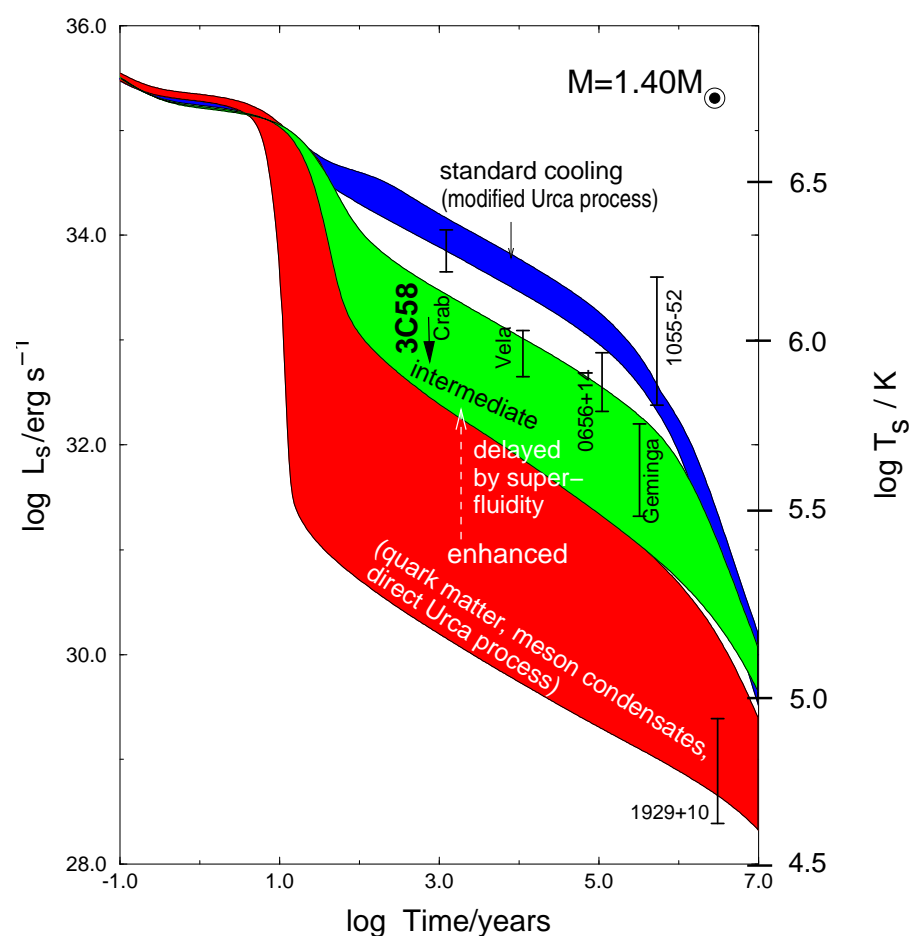

Figure 13: Cooling behavior of a $1.4 M_{\odot}$ neutron star for competing assumptions about the properties of superdense matter. Three distinct cooling scenarios, referred to as standard, intermediate, and enhanced can be distinguished. The band-like structures reflect the uncertainties inherent in the stellar EoS [2, 4].

neutron stars immediately after formation is neutrino emission, with an initial cooling time scale of seconds. Already a few minutes after birth, the internal neutron star temperature drops to $\sim 10^{9} \mathrm{~K}$. Photon emission overtakes neutrino emission when the internal temperature has fallen to $\sim 10^{8} \mathrm{~K}$, with a corresponding surface temperature roughly two orders of magnitude smaller. Neutrino cooling dominates for at least the first $10^{3}$ years, and typically for much longer in standard cooling (modified Urca) calculations. The dominant neutrino emitting processes in neutron star matter are summarized in Tables 2 and 3. Figure 13 shows the outcome of cooling calculations performed for a broad collection of equations of state $[2,4]$ and competing assumptions about the dominant neutrino emitting processes. For recent overviews of neutron star cooling, see, for instance, Refs. [21, 98]. We also refer to D. Blaschke's contribution contained elsewhere in this volume.

\section{Summary}

It is often stressed that there has never been a more exciting time in the overlapping areas of nuclear physics, particle physics and relativistic astrophysics than today. This comes at a time where new orbiting observatories such as the Hubble Space Telescope (HST), Rossi X-ray Timing Explorer, Chandra X-ray satellite, and the X-ray Multi Mirror Mission (XMM) have extended our vision tremendously, allowing us to observe compact star phenomena with an unprecedented clarity and angular resolution that previously were only imagined. On the Earth, radio telescopes (Arecibo, Green Bank, Parkes, VLA) and instruments using adaptive optics and other revolutionary 
techniques have exceeded previous expectations of what can be accomplished from the ground. Finally, the gravitational wave detectors LIGO, LISA, and VIRGO are opening up a window for the detection of gravitational waves emitted from compact stellar objects such as neutron stars and black holes. This unprecedented situation is providing us with key information on compact stars, which are the only physical objects in which cold and dense baryonic matter is realized in nature. As discussed in this paper, a key role in compact star physics is played by strangeness. It alters the masses, radii, cooling behavior, and surface composition of neutron stars. Other important observables may be the spin evolution of isolated neutron stars and neutron stars in low-mass x-ray binaries. All told, these observables are key in exploring the phase diagram of dense nuclear matter at high baryon number density but low temperature, which is not accessible to relativistic heavy ion collision experiments.

\section{References}

[1] N. K. Glendenning, Compact Stars, Nuclear Physics, Particle Physics, and General Relativity, 2nd ed. (Springer-Verlag, New York, 2000).

[2] F. Weber, Pulsars as Astrophysical Laboratories for Nuclear and Particle Physics, High Energy Physics, Cosmology and Gravitation Series (IOP Publishing, Bristol, Great Britain, 1999).

[3] J. M. Lattimer and M. Prakash, Astrophys. J. 550 (2001) 426.

[4] F. Weber, Prog. Part. Nucl. Phys. 54 (2005) 193, (astro-ph/ 0407155 ).

[5] K. Rajagopal and F. Wilczek, The Condensed Matter Physics of QCD, At the Frontier of Particle Physics / Handbook of QCD, ed. M. Shifman, (World Scientific) (2001).

[6] M. Alford, Ann. Rev. Nucl. Part. Sci. 51 (2001) 131.

[7] S. B. Ruster, V. Werth, M. Buballa, I. A. Shovkovy, and D. H. Rischke, Phys. Rev. D 72 (2005) 034004 .

[8] C. Alcock, E. Farhi, and A. V. Olinto, Astrophys. J. 310 (1986) 261.

[9] C. Alcock and A. V. Olinto, Ann. Rev. Nucl. Part. Sci. 38 (1988) 161.

[10] J. Madsen, Lecture Notes in Physics 516 (1999) 162.

[11] N. K. Glendenning, Astrophys. J. 293 (1985) 470.

[12] M. Alford and S. Reddy, Phys. Rev. D 67 (2003) 074024.

[13] H. Lenske and C. Fuchs, Phys. Lett. 345B (1995) 355.

[14] F. Hofmann, C. M. Keil, and H. Lenske, Phys. Rev. C 64 (2001) 034314.

[15] H. Huber, F. Weber, M. K. Weigel, and Ch. Schaab, Int. J. Mod. Phys. E 7, No. 3 (1998) 301.

[16] M. Prakash, I. Bombaci, M. Prakash, P. J. Ellis, J. M. Lattimer, and R. Knorren, Phys. Rep. 280 (1997) 1.

[17] J. M. Lattimer, C. J. Pethick, M. Prakash, and P. Haensel, Phys. Rev. Lett. 66 (1991) 2701.

[18] M. Prakash, M. Prakash, J. M. Lattimer, and C. J. Pethick, Astrophys. J. 390 (1992) L77.

[19] P. Haensel and O. Yu. Gnedin, Astron. \& Astrophys. 290 (1994) 458.

[20] Ch. Schaab, F. Weber, M. K. Weigel, and N. K. Glendenning, Nucl. Phys. A605 (1996) 531. 
[21] D. Page, U. Geppert, and F. Weber, Neutron Star Cooling, Nuclear Physics A (in press), (astro-ph/0508056).

[22] T. Waas, M. Rho, and W. Weise, Nucl. Phys. A617 (1997) 449.

[23] N. K. Glendenning and J. Schaffner-Bielich, Phys. Rev. C 60 (1999) 025803.

[24] G. Mao, P. Papazoglou, S. Hofmann, S. Schramm, H. Stöcker, and W. Greiner, Phys. Rev. C 59 (1999) 3381.

[25] G. E. Brown, Supernova Explosions, Black Holes and Nucleon Stars, in: Proceedings of the Nuclear Physics Conference - INPC '95, ed. by S. Zuxun and X. Jincheng (World Scientific, Singapore, 1996) p. 623 .

[26] G. Q. Li, C.-H. Lee, and G. E. Brown, Nucl. Phys. A625 (1997) 372.

[27] G. Q. Li, C.-H. Lee, and G. E. Brown, Phys. Rev. Lett. 79 (1997) 5214.

[28] G. E. Brown, Phys. Bl. 53 (1997) 671.

[29] R. L. Jaffe, Phys. Lett. 38 (1977) 195.

[30] R. Tamagaki, Prog. Theor. Phys. 85 (1991) 321.

[31] T. Sakai, J. Mori, A. J. Buchmann, K. Shimizu, and K. Yazaki, Nucl. Phys. A625 (1997) 192.

[32] N. K. Glendenning and J. Schaffner-Bielich, Phys. Rev. C 58 (1998) 1298.

[33] A. Faessler, A. J. Buchmann, M. I. Krivoruchenko, and B. V. Martemyanov, Phys. Lett. 391B (1997) 255.

[34] A. Faessler, A. J. Buchmann, and M. I. Krivoruchenko, Phys. Rev. C 56 (1997) 1576.

[35] N. K. Glendenning, Phys. Rev. D 46 (1992) 1274.

[36] F. Weber, J. Phys. G: Nucl. Part. Phys. 25 (1999) R195.

[37] N. K. Glendenning and F. Weber, Signal of Quark Deconfinement in Millisecond Pulsars and Reconfinement in Accreting X-ray Neutron Stars, Lecture Notes in Physics 578, (Springer-Verlag, Berlin, 2001), p. 305.

[38] M. Alford, K. Rajagopal, and F. Wilczek, Phys. Lett. 422B (1998) 247.

[39] R. Rapp, T. Schäfer, E. V. Shuryak, and M. Velkovsky, Phys. Rev. Lett. 81 (1998) 53; Ann. Phys. 280 (2000) 35 .

[40] M. Alford, C. Kouvaris, and K. Rajagopal, Phys. Rev. Lett. 92 (2004) 222001.

[41] K. Rajagopal and F. Wilczek, Phys. Rev. Lett. 86 (2001) 3492.

[42] P. F. Bedaque and T. Schäfer, Nucl. Phys. A697 (2002) 802.

[43] D. B. Kaplan and S. Reddy, Phys. Rev. D 65 (2002) 054042.

[44] M. Buballa, J. Hosek and M. Oertel, Phys. Rev. Lett. 90 (2003) 182002.

[45] A. Schmitt, Phys. Rev. D 71 (2004) 054016, (nucl-th/ 0412033 ).

[46] T. Schäfer, Phys. Rev. D 62 (2000) 094007.

[47] M. Alford, J. A. Bowers, and K. Rajagopal, Phys. Rev. D 63 (2001) 074016.

[48] J. A. Bowers and K. Rajagopal, Phys. Rev. D 66 (2002) 065002. 
[49] R. Casalbuoni and G. Nardulli, Rev. Mod. Phys. 76 (2004) 263.

[50] M. Alford, K. Rajagopal, and F. Wilczek, Nucl. Phys. B537 (1999) 443.

[51] D. T. Son, Phys. Rev. D D59 (1999) 094019.

[52] M. Alford, J. Phys. G 30 (2004) S441.

[53] K. Rajagopal, Acta Physica Polonica B 31 (2000) 3021.

[54] M. Alford, J. A. Bowers, and K. Rajagopal, J. Phys. G 27 (2001) 541.

[55] D. Blaschke, D. M. Sedrakian, and K. M. Shahabasyan, Astron. \& Astrophys. 350 (1999) L47.

[56] The AMS home page is http://ams.cern.ch.

[57] Information about ECCO can be found at http: / / ultraman . berkeley .edu.

[58] J. Madsen, Phys. Rev. Lett. 87 (2001) 172003.

[59] Strange Quark Matter in Physics and Astrophysics, Proc. of the International Workshop, ed. by J. Madsen and P. Haensel, Nucl. Phys. B (Proc. Suppl.) 24B (1991).

[60] A. R. Bodmer, Phys. Rev. D 4 (1971) 1601.

[61] E. Witten, Phys. Rev. D 30 (1984) 272.

[62] H. Terazawa, INS-Report-338 (INS, Univ. of Tokyo, 1979); J. Phys. Soc. Japan, 58 (1989) 3555; 58 (1989) 4388; 59 (1990) 1199.

[63] J. Madsen, Phys. Rev. Lett. 61 (1988) 2909.

[64] J. Madsen, Physics and Astrophysics of Strange Quark Matter, Proc. of the 2nd International Conference on Physics and Astrophysics of Quark-Gluon Plasma, ed. by B. Sinha, Y. P. Viyogi, and S. Raha (World Scientific, Singapore, 1994) p. 186.

[65] Ch. Kettner, F. Weber, M. K. Weigel, and N. K. Glendenning, Phys. Rev. D 51 (1995) 1440.

[66] N. K. Glendenning and F. Weber, Astrophys. J. 400 (1992) 647.

[67] M. Stejner and J. Madsen, Phys. Rev. D 72 (2005) 123005.

[68] P. Jaikumar, S. Reddy, A. W. Steiner, The Strange Star Surface: A Crust with Nuggets, (nucl-th/0507055).

[69] K. S. Thorne, in: Proc. Int. School of Phys. "Enrico Fermi”, Course 35, High Energy Astrophysics, ed. by L. Gratton (Academic Press, New York, 1966) p. 166.

[70] R. C. Tolman, Phys. Rev. 55 (1939) 364.

[71] J. R. Oppenheimer and G. M. Volkoff, Phys. Rev. 55 (1939) 374.

[72] D. J. Nice, E. M. Splaver, and I. H. Stairs, in: Binary Radio Pulsars, Eds. F. A. Rasio and I. H. Stairs, ASP Conference Series, (2005), (astro-ph/ 00411207$).$

[73] H. Quaintrell et al., Astron. \& Astrophys. 401 (2003) 313.

[74] J. A. Orosz and E. Kuulkers, Mon. Not. R. Astron. Soc. 305 (1999) 132.

[75] L. Titarchuck and N. Shaposhnikov, Astrophys. J. 570 (2002) L25.

[76] G. E. Brown and H. A. Bethe, Astrophys. J. 423 (1994) 659.

[77] H. A. Bethe and G. E. Brown, Astrophys. J. 445 (1995) L129. 
[78] J. L. Friedman, J. R. Ipser, and L. Parker, Astrophys. J. 304 (1986) 115.

[79] J. B. Hartle, Astrophys. J. 150 (1967) 1005.

[80] M. Bejger and P. Haensel, Astron. \& Astrophys. 420 (2004) 987.

[81] N. K. Glendenning, Phys. Rev. D 46 (1992) 4161.

[82] L. Lindblom and B. Owen, Phys. Rev. D 65 (2002) 063006.

[83] L. Lindblom, Neutron Star Pulsations and Instabilities, in: Gravitational Waves: A Challenge to Theoretical Astrophysics, edited by V. Ferrari, J. C. Miller, and L. Rezzolla, ICTP Lecture Notes Series, Vol. III, (ISBN 92-95003-05-5, May 2001), (ast ro-ph/0101136).

[84] J. Madsen, Phys. Rev. Lett. 81 (1998) 3311.

[85] J. Madsen, Phys. Rev. Lett. 85 (2000) 10.

[86] M. van der Klis, Ann. Rev. Astron. Astrophys. 38 (2000) 717.

[87] C. Manuel, A. Dobado, and F. J. Llanes-Estrada, Shear Viscosity in a CFL Quark Star, (hep-ph/0406058).

[88] N. K. Glendenning, S. Pei, and F. Weber, Phys. Rev. Lett. 79 (1997) 1603.

[89] N.K. Spyrou and N. Stergioulas, Astron. \& Astrophys. 395 (2002) 151.

[90] N. K. Glendenning and S. Pei, Phys. Rev. C 52 (1995) 2250.

[91] J. L. Zdunik, P. Haensel, E. Gourgoulhon, and M. Bejger, Astron. \& Astrophys. 416 (2004) 1013.

[92] N. K. Glendenning and F. Weber, Astrophys. J. 559 (2001) L119.

[93] L. Bildsten, Astrophys. J. 501 (1998) L89.

[94] N. Andersson, D. I. Jones, K. D. Kokkotas, and N. Stergioulas, Astrophys. J. 534 (2000) L75.

[95] D. Chakrabarty et al., Nature 424 (2003) 42.

[96] E. Chubarian, H. Grigorian, G. Poghosyan, and D. Blaschke, Astron. \& Astrophys. 357 (2000) 968.

[97] G. Poghosyan, H. Grigorian, D. Blaschke, Astrophys. J. 551 (2001) L73.

[98] D. G. Yakovlev and C. J. Pethick, Ann. Rev. Astron. Astrophys. 42 (2004) 169, (astro-ph/0402143). 